\title{
Herculanum. Des archives aux restitutions architecturales et décoratives
}

Campagne 2015 du projet ANR VESUVIA

Alexandra Dardenay, Agnes Allroggen-Bedel, Hélène Eristov, Adeline Grand-Clément, Marie-Laure Maraval, Carla Marotta, Nicolas Monteix et Emmanuelle Rosso

\section{(2) OpenEdition Journals}

Édition électronique

URL : http://journals.openedition.org/cefr/1588

DOI : $10.4000 /$ cefr. 1588

ISSN : 2282-5703

Éditeur

École française de Rome

Référence électronique

Alexandra Dardenay, Agnes Allroggen-Bedel, Hélène Eristov, Adeline Grand-Clément, Marie-Laure Maraval, Carla Marotta, Nicolas Monteix et Emmanuelle Rosso, « Herculanum. Des archives aux restitutions architecturales et décoratives ", Chronique des activités archéologiques de l'École française de Rome [En ligne], Les cités vésuviennes, mis en ligne le 30 juin 2016, consulté le 02 mai 2019. URL http://journals.openedition.org/cefr/1588; DOI : 10.4000/cefr.1588

Ce document a été généré automatiquement le 2 mai 2019.

(c) École française de Rome 


\title{
Herculanum. Des archives aux restitutions architecturales et décoratives
}

Campagne 2015 du projet ANR VESUVIA

\author{
Alexandra Dardenay, Agnes Allroggen-Bedel, Hélène Eristov, Adeline \\ Grand-Clément, Marie-Laure Maraval, Carla Marotta, Nicolas Monteix et \\ Emmanuelle Rosso
}

\section{NOTE DE L'ÉDITEUR}

Notre profonde reconnaissance est adressée aux institutions apportant leur collaboration au projet VESUVIA.

\section{NOTE DE L'AUTEUR}

Avec la collaboration de M. Carrive, et des membres d'Archéovision (J.-Bernard, L.

Espinasse, P. Mora)

1 La campagne de 2015 du programme VESUVIA (Habitat et Société à Herculanum) s'est caractérisée par la multiplication des enquêtes archivistiques et muséales parallèlement aux travaux de terrain. En effet, l'enregistrement des décors in situ étant pratiquement achevé, l'attention de l'équipe se concentre désormais sur la recontextualisation des éléments prélevés lors des différentes phases de fouilles du site et sur la réalisation d'infographies de restitution à projeter dans les modèles 3D (fig. 1). 
Fig. 1 - Cartographie des différentes phases de fouilles sur le site d'Herculanum.

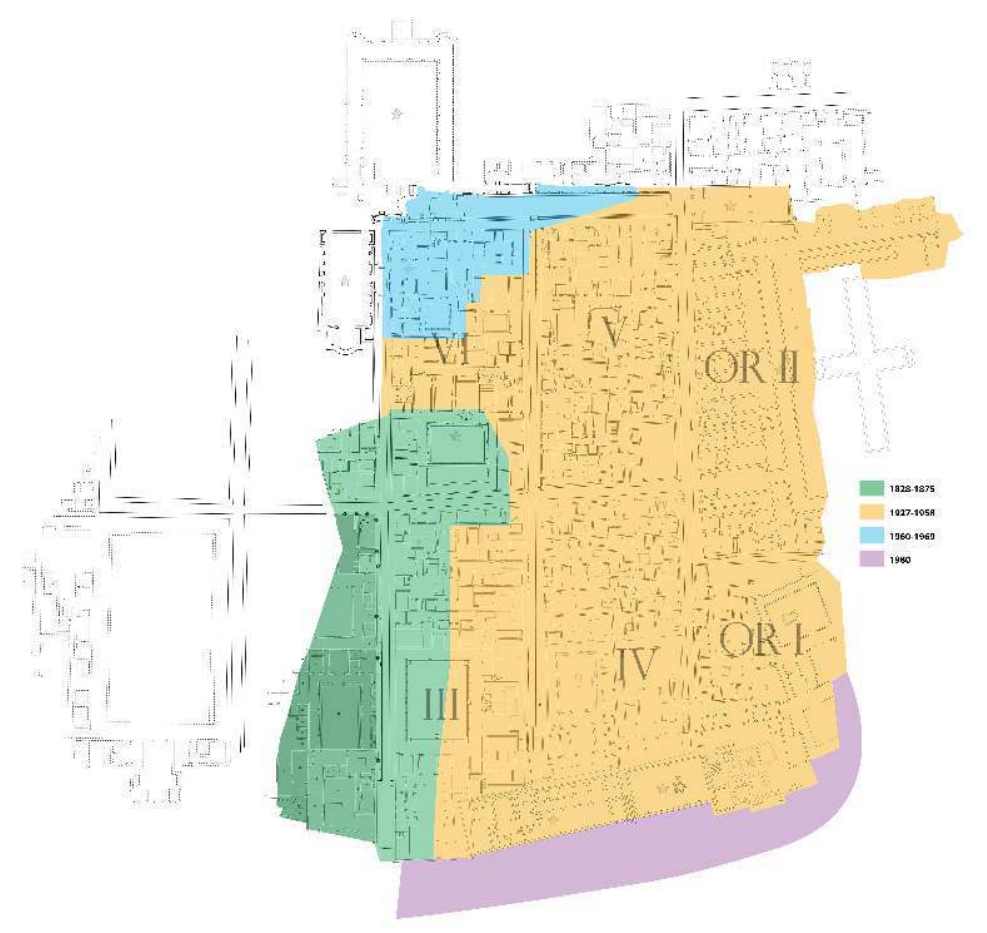

DAO MARIE LAURE MARAVAL. AVEC L'AIMABLE AUTORISATION DE LA SOPRINTENDENZA ET DU MINISTERO DEI BENI E DELLE ATTIVITÀ CULTURALI E DEL TURISMO.

Dans le même temps, nos collaborateurs d'Archéovision (UMS SHS 3D nº3657: JeanFrançois Bernard, Loïc Espinasse et Pascal Mora) ont pu proposer une première version des cinq édifices sélectionnés pour un modèle 3D en raison de leur potentiel ${ }^{1}$. À la suite de campagnes d'acquisition photographique in situ, les ingénieurs d'Archéovision sont actuellement en phase de modélisation des maisons. Parallèlement, ils réalisent des orthophotographies des parois, afin de permettre des restitutions infographiques des décors dans leur état originel. Ces infographies offrent une restauration graphique des parties détruites et/ou prélevées et seront ensuite intégrées dans les modèles 3D des édifices, à l'instar des éléments de mobilier dont le contexte de découverte a pu être établi, et qui ont également été modélisés.

Pour une meilleure visibilité du travail accompli et des structures étudiées, ils ont positionné une version axonométrique de ces édifices sur le plan d'Herculanum réalisé par Marie-Laure Maraval dans le cadre du programme VESUVIA (fig. 2). 
Fig. 2 - Axonométrie de quelques édifices d'Herculanum.

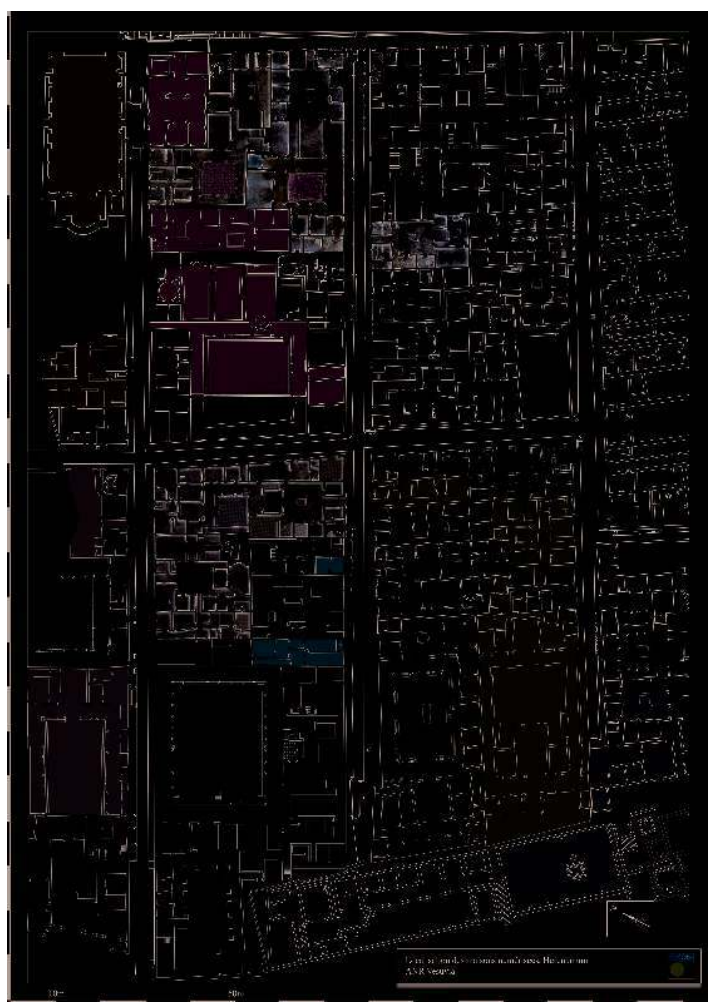

Casa di Nettuno ed Anfitrite (insula V), Casa del colonnato tuscanico et Casa del salone nero (insula VI), Casa del tramezzo di legno et Casa dello scheletro (insula III).

RÉALISATION : ARCHÉOVISION. AVEC L'AIMABLE AUTORISATION DE LA SOPRINTENDENZA ET DU MINISTERO DEI BENI E DELLE ATTIVITÀ CULTURALI E DEL TURISMO.

Du point de vue méthodologique, l'approche est double. L'enquête dans les archives et dans les réserves du musée de Naples se mène naturellement à l'échelle du site, l'objectif étant de rassembler l'ensemble de la documentation disponible, quelle que soit sa nature (journaux de fouilles, correspondance scientifique, enregistrement de mobilier, inventaires etc...) pour reconstituer l'histoire des édifices depuis leur mise au jour mais également recontextualiser les œuvres et éléments de mobilier. Dans ce type d'enquête, nous ne ciblons pas forcément un édifice en particulier puisque nous procédons par fonds et types de sources, mais une telle approche est néanmoins possible, notamment pour les édifices fouillés au XIX siècle, comme la Casa d'Argo par exemple. L'étude est donc différente du travail sur le terrain qui nécessite, quant à lui, un enregistrement des données par édifice en priorité. C'est la combinaison des différents résultats, informatisés à travers la base de données DOMVS@ du projet, qui permet d'avancer vers les synthèses architecturales et ornementales. Ces dernières donneront lieu, dans une dernière phase du programme, à une lecture anthropologique et sociale.

5 C'est ainsi que parallèlement aux explorations des fonds d'archives, les recherches en 2015 se sont notamment concentrées sur trois édifices. La Casa di Nettuno ed Anfitrite, à propos de laquelle les premiers résultats en matière de restitution infographique du triclinium 7 ont été présentés dans une précédente chronique ${ }^{2}$, a continué de concentrer les travaux de l'équipe du programme. La Casa del salone nero a, d'autre part, fait l'objet d'une étude monographique menée par Iris Pingeon, étudiante de Master 2 en Sciences de l'Antiquité à l'Université de Toulouse $\mathrm{II}^{3}$ qui a focalisé ses recherches sur l'histoire 
architecturale de l'édifice - à travers des études du bâti - et l'identification d'éléments de décor prélevés. Par ailleurs, l'entrée en doctorat d'une jeune chercheuse italienne, Carla Marotta, avec un sujet de thèse portant sur la Casa d'Argo', a permis de développer le volet du programme consacré aux fouilles du XIX ${ }^{\mathrm{e}}$ siècle.

\section{Les archives au service des reconstitutions infographiques : un cas emblématique, la Casa d'Argo}

Les tentatives de reconstitution du programme décoratif des demeures d'Herculanum nécessitent de recourir à des fonds d'archives très variés, liés à l'histoire de la mise en valeur du site et de sa fréquentation, depuis sa découverte au XVIII ${ }^{\mathrm{e}}$ siècle : carnets et journaux de fouilles, inventaires de musées, mais aussi correspondance scientifique, archives graphiques et photographiques, journaux de voyageurs, registres de visite... C'est souvent le seul moyen de restituer l'emplacement et l'aspect d'origine des peintures qui, dans leur grande majorité, ont été transférées au Museo archeologico nazionale di Napoli (MANN), se sont dégradées ou ont tout simplement disparu. La précédente chronique insistait sur l'apport significatif des données archivistiques pour la reconstitution des peintures du triclinium de la Casa di Nettuno ed Anfitrite, fouillée par A. Maiuri. Or le dépouillement des archives s'avère encore plus nécessaire dans le cas de maisons dégagées au XIX ${ }^{e}$ siècle, qui ont subi entre 1875 et nos jours d'importantes dégradations et n'ont conservé in situ que peu de traces de leur décor d'origine. C'est le cas en particulier de la Casa d'Argo, située dans l'insula II, qui possédait deux étages, aujourd'hui effondrés (fig. 3).

Fig. 3 - Casa d'Argo, vue du péristyle.

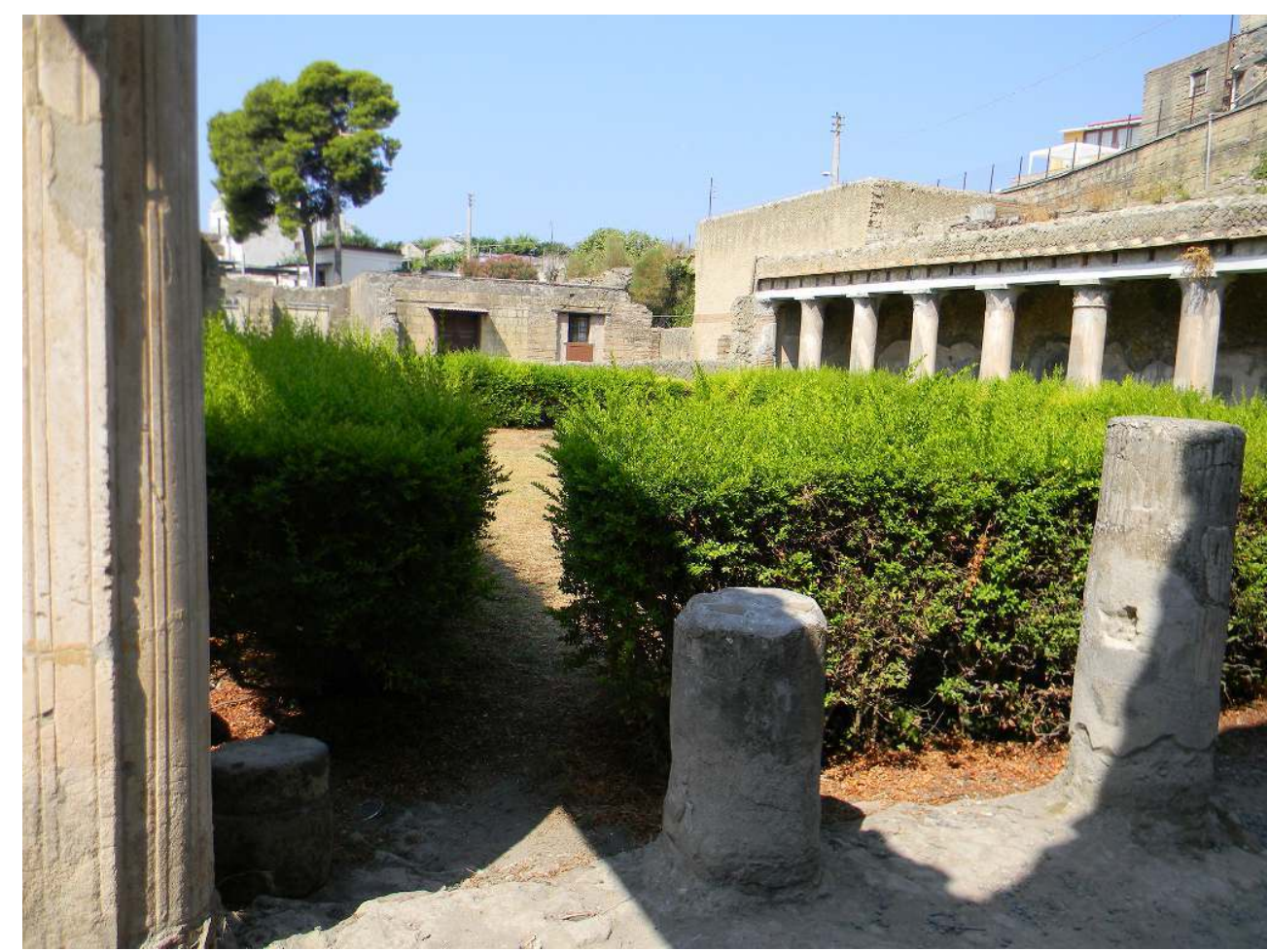

Cliché C. Marotta. Avec l'aimable autorisation de la Soprintendenza et du Ministero dei Beni e delle Attività Culturali e del Turismo. 
La casa d'Argo est la première maison d'Herculanum à avoir été entièrement dégagée, lorsque commencent les fouilles à ciel ouvert, sous la direction de l'architecte Carlo Bonucci, à partir de janvier 1828. Les journaux de fouille des années 1828-1830 (édités par M. Ruggiero ${ }^{5}$ ) nous permettent de suivre la nature des découvertes effectuées au fur et à mesure de la progression des fouilles. L'habitation a reçu son nom d'une peinture aujourd'hui perdue, qui a été découverte en décembre 1828, dans le triclinium sud donnant sur le péristyle. On y voyait le dieu Mercure tentant d'endormir le géant Argus pour enlever Io $^{6}$. Lorsque les étages, puis le rez-de-chaussée de la maison ont été dégagés entre janvier 1828 et août 1830, certaines peintures manquaient déjà : C. Bonucci a relevé en effet des traces de tunnels du XVIII ${ }^{\mathrm{e}}$ siècle et constaté le prélèvement de certains tableaux, en particulier au niveau du portique. D'après les travaux de recoupement effectués par Agnès Allroggen-Bedel, les cunicoli des Bourbons auraient atteint cette demeure autour de 1745.

8 La présence des fouilleurs du XVIII ${ }^{\mathrm{e}}$ siècle dans la Casa d'Argo peut être déduite de la comparaison entre quelques panneaux de peinture conservés au Musée de Naples et les vestiges de décors pariétaux demeurés in situ. Dans le musée se trouve un beau fragment à fond blanc présentant la partie supérieure d'un édicule et une tenture de couleur bleue, ourlée d'une guirlande de vigne (MANN 9863)7 (fig. 4).

Fig. 4 - Panneau de peinture, provenant de la Casa d'Argo, pièce 17.

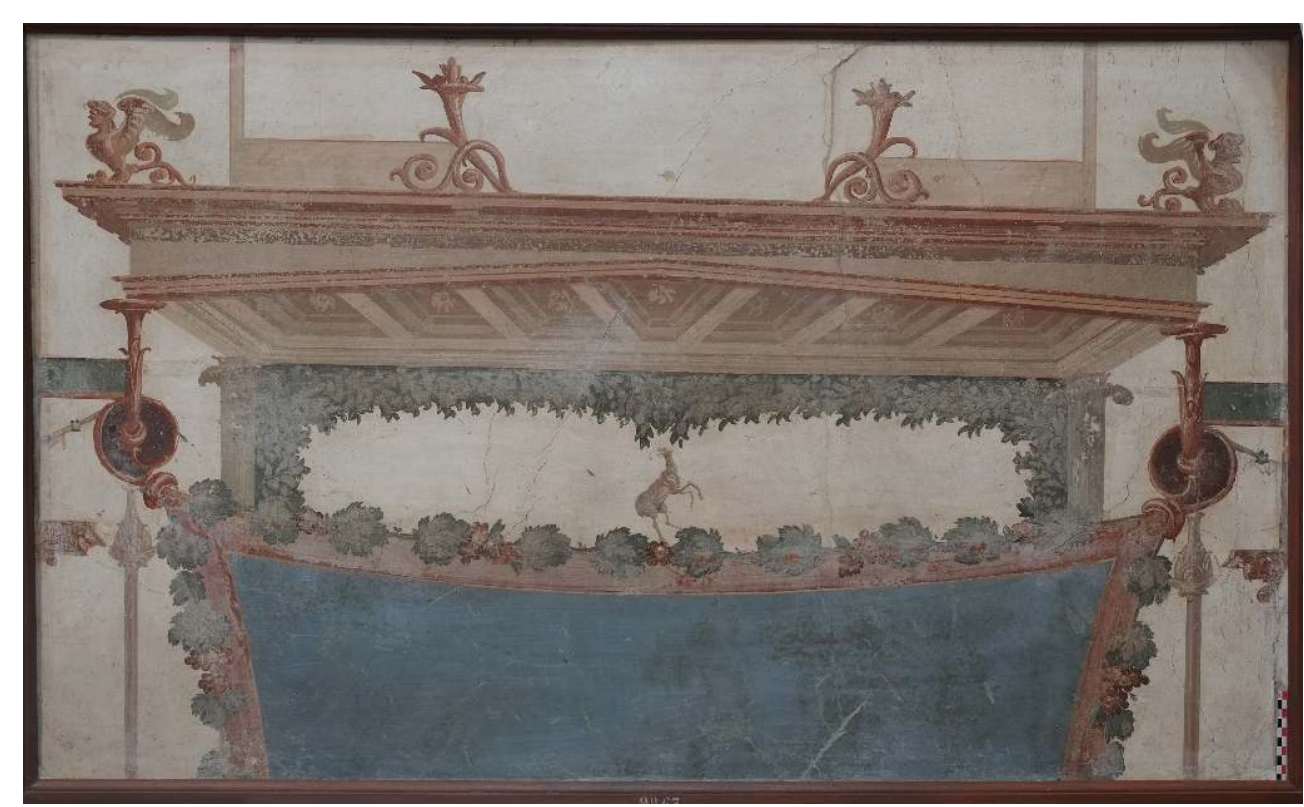

MANN, inv. n॰9863; Cliché M.-L. Maraval. Avec l'aimable autorisation de la Soprintendenza et du Ministero dei Beni e delle Attività Culturali e del Turismo.

Dans la pièce 17 de la Casa d'Argo ${ }^{8}$, on distingue encore les vestiges d'un décor qui montre les mêmes motifs, permettant ainsi l'attribution du fragment du musée à la zone supérieure de cette pièce. La tenture ourlée de la guirlande de vigne correspond à la description d'une peinture, rapportée par J. Alcubierre le 16 et le 19 octobre 1745 : «Un leño enlazado de hojas de Parro, y uvas ${ }^{9}$. Les restes de décor in situ, la peinture du musée et la mention de J. Alcubierre confirment donc la présence des fouilleurs des Bourbons dans la Casa d'Argo entre le 16 et le 19 octobre 1745. De la même pièce proviennent des peintures avec paons sur une sorte de trépied (MANN 8759). Sur le plus grand fragment, à

Chronique des activités archéologiques de l'École française de Rome, Les cités vésuviennes | 2016 
gauche, les traces d'un sarment de vigne sont visibles, qui correspondent parfaitement à celui qui couronne la tenture bleue de l'autre fragment. D'après la composition des peintures in situ, les deux fragments se trouvaient de part et d'autre de l'édicule central.

Dans une autre pièce de la même maison furent détachés deux fragments figurant des masques tragiques (MANN 9821). Les pendants sont restés dans la pièce 20 , permettant ainsi de localiser les deux fragments, détachés de la zone supérieure de la paroi $\operatorname{sud}^{10}$. La date de leur découverte n'est pas connue. D'après M. Pagano et R. Prisciandaro, elles doivent être identifiées parmi les 15 peintures répertoriées dans une annexe à une lettre du 13 septembre $1745^{11}$. À cet emplacement sont mentionnées, en effet, deux peintures avec masques, rassemblées dans une des 12 « casse ». Il s'agit de "quattro mascheroni di lunghezza pal. 2 oncie 7, e d'altezza oncie 8 ", dont les mesures, cependant, ne correspondent pas aux deux fragments provenant de la Casa d'Argo ${ }^{12}$. L'inventaire ne porte pas de date, mais la lettre d'accompagnement offre un terminus ante quem pour la découverte des peintures ${ }^{13}$. Dans les rapports de J. Alcubierre de cette période, on ne trouve pas de représentation de masques. En septembre 1745, les fouilleurs travaillent en effet à détacher une grande quantité de peintures appartenant à un bel ensemble de III ${ }^{\mathrm{e}}$ style, dont nous savons seulement qu'il se trouvait non loin du théâtre ${ }^{14}$.

11 La mission effectuée à Naples en septembre 2015 a permis d'examiner une partie des archives de la Soprintendenza archeologica della Campania - sede di Napoli relatives à la Casa d'Argo, afin de rassembler de la documentation iconographique et textuelle sur le décor aujourd'hui disparu et de proposer d'autres rattachements de fresques ou d'objets conservés au MANN.

12 Malheureusement, les informations trouvées à l'Archivio disegni se sont avérées décevantes, car une bonne part du fonds documentaire a été transférée aux archives de la Soprintendenza archeologica di Pompei après sa création en 1981. À l'intérieur du maigre dossier Ercolano $R A / 10$ on a toutefois retrouvé deux reproductions graphiques du tableau figurant « Persée tuant Méduse à l'aide de Minerve ». La première, une aquarelle datée du 11 septembre 1828, porte la signature de C. Bonucci; la seconde, un dessin au crayon exécuté en 1829, est de la main de Giuseppe Marsigli, un peintre qui a collaboré à la réalisation des planches du Real Museo Borbonico. On peut ainsi confronter ces précieux témoignages iconographiques à la brève description de la peinture fournie par Bonucci, lorsqu'elle a été découverte en décembre 1828 : «Nella stanza numero 14 si scorse un bel quadro rappresentante Perseo che uccide Medusa, guardandosi nello scudo di Minerva che gli era vicina e che brandisce contro della vinta la sua terribile lancia. Nel fondo di questa dipintura si osserva da un lato una porta, alcune torri e delle fortificazioni, e dall'altro una campagna, degli alberi e qualche armento $»^{15}$. L'aquarelle, en particulier, a le mérite de fournir quelques indications sur les couleurs utilisées, que le fouilleur passe totalement sous silence: on apprend notamment que Persée portait un manteau bleu (fig. 5). 
Fig. 5 - « Persée tuant Méduse à l'aide de Minerve ».

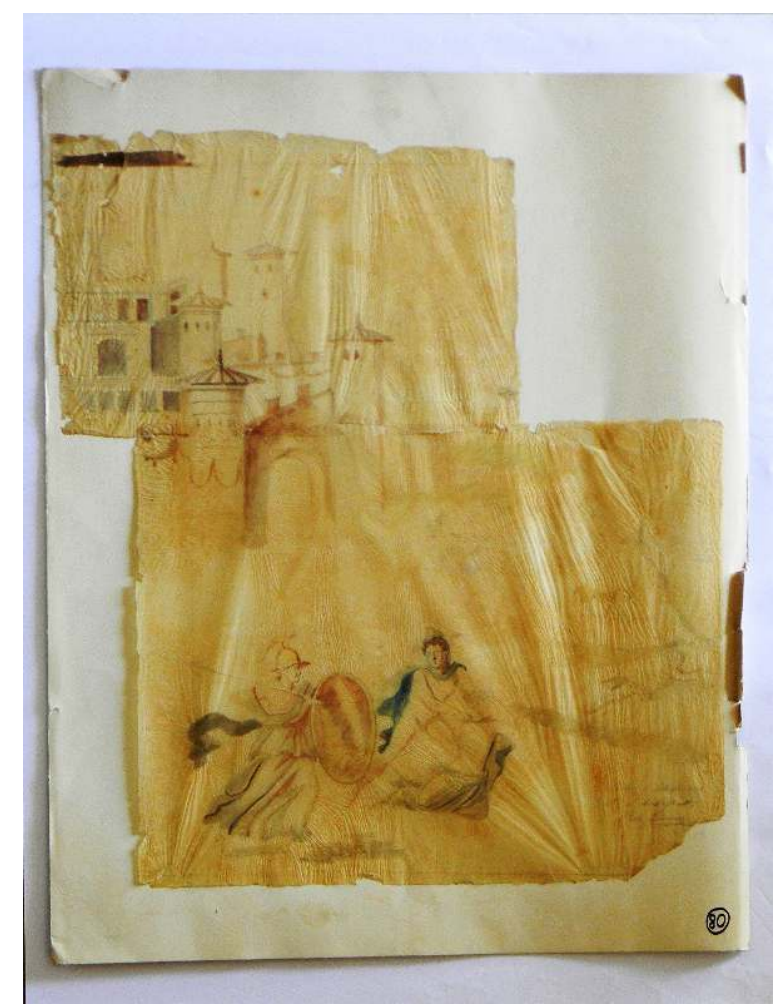

\section{AQUARELLE RÉALISÉE EN 1828.}

Cartella Ercolano RA/10 n. 5530, Archivio disegni della Soprintendenza per i Beni archeologici di Napoli. Avec l'aimable autorisation de la Soprintendenza et du Ministero dei Beni e delle Attività Culturali e del Turismo.

Par ailleurs, les recherches menées dans les archives photographiques de la Surintendance n'ont pas été concluantes. En effet, l'organisation des dossiers rend difficile l'identification des fresques et des objets photographiés, tandis que l'indication de la provenance de la majorité d'entre eux reste inconnue ou imprécise. Nous n'avons ainsi trouvé aucune photographie pouvant correspondre à l'une des fresques qui décoraient la maison, en particulier la scène représentant Io et Argus. Malgré tout, le croisement des rapports de fouilles de $\mathrm{C}$. Bonucci avec certaines photographies d'archives a permis de rattacher à la Casa d'Argo trois objets dont la provenance était jusqu'ici inconnue : un bracelet serpentiforme en or trouvé le 10 mars 1828 (MANN, inv. $n^{\circ} 25622$ ) et deux appliques en argent trouvées en octobre 1828, l'une ornée du buste de Diane (MANN, inv. ${ }^{\circ}$ 25495) et l'autre du buste d'Apollon (fig. 6 ; MANN, inv. ${ }^{\circ} 25492$ ). 
Fig. 6 - Casa d'Argo, buste d'Apollon, applique en argent.

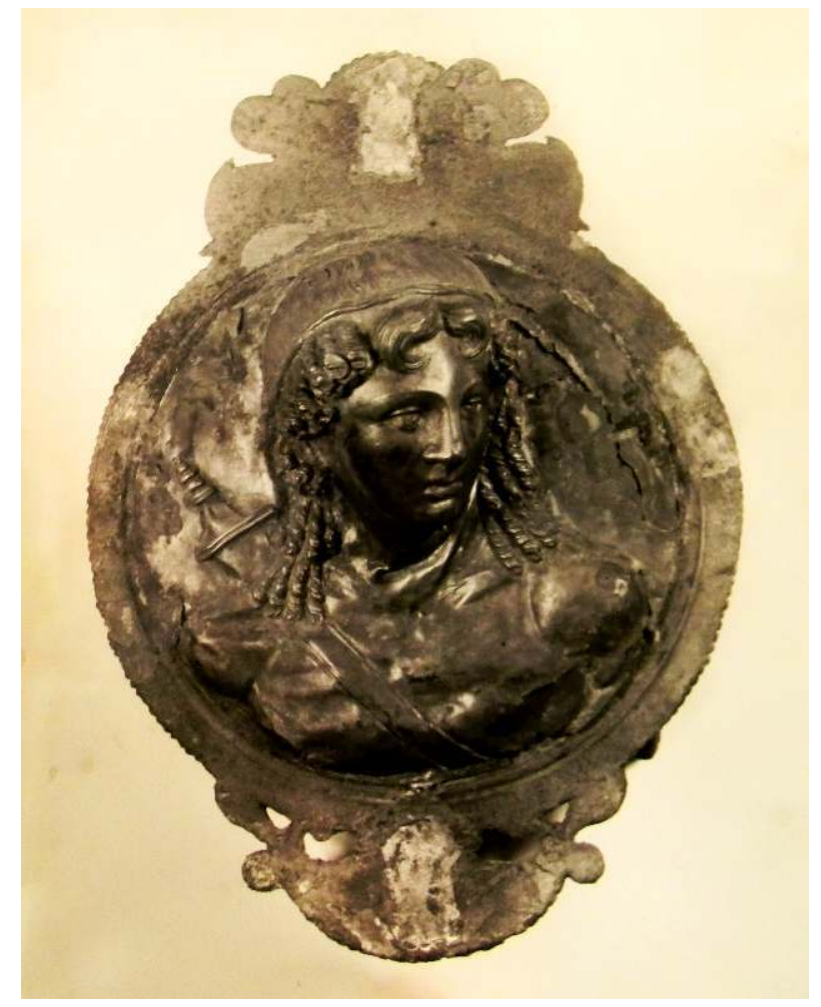

MANN, inv. n 25493. Avec l'aimable autorisation de la Soprintendenza et du Ministero dei Beni e delle Attività Culturali e del Turismo.

Les résultats les plus significatifs viennent donc de l'Archivio storico. La correspondance concernant les années suivant la découverte de la maison a fourni, par exemple, des indications précieuses quant au destin de l'un des tableaux les plus remarquables de la Casa d'Argo : il s'agit d'une peinture représentant Persée en train de trancher la tête de Méduse. Cette peinture pouvait se trouver dans la pièce 23, selon la numérotation du plan actuel. Une lettre envoyée à Michele Arditi, directeur du Musée et Surintendant des fouilles par le marquis de Ruffo, ministre de la Maison Royale, le 15 juin 1829, indique que le tableau a été transféré au Musée royal sur ordre du roi (qui avait visité en personne le chantier peu de temps auparavant $)^{16}$. Or on n'en retrouve pas de trace au MANN aujourd'hui, alors qu'il était bien visible en 1839, si l'on en croit la publication de G. Finati ${ }^{17}$. De plus, les rapports des fouilles de $\mathrm{C}$. Bonucci étaient accompagnés du plan d'une pièce située à l'étage supérieur, apparemment utilisée comme placard, et d'un dessin du décor de la paroi gauche de cette pièce (fig. 7). 
Fig. 7 - Casa d'Argo, dessin du décor d'une paroi de l'étage supérieur.

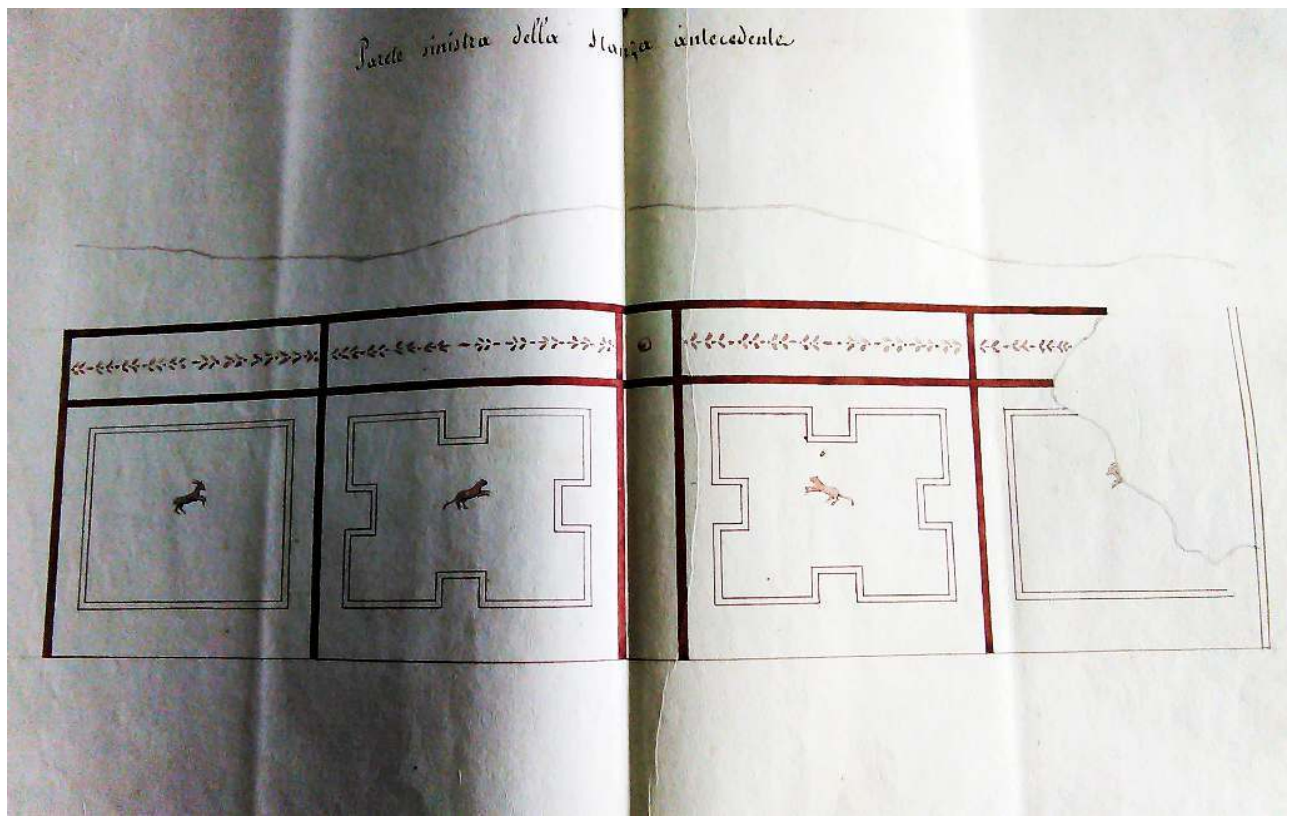

Archivio storico della Soprintendenza per i Beni archeologici di Napoli ; fasc. VII C9, 3. Avec l'aimable autorisation de la Soprintendenza et du Ministero dei Beni e delle Attività Culturali e del Turismo.

Une autre découverte, fortuite, a été faite au milieu du dossier B.145 folio 5, Ercolano, descrizioni di ambienti: 1931-1938, dossier qui contient les descriptions de pièces d'autres maisons d'Herculanum. Il s'agit d'un papier-calque qui fournit un plan de la Casa d'Argo à l'échelle $1: 4000$, réalisé à partir d'un original qui pourrait bien être celui - aujourd'hui perdu - réalisé par C. Bonucci lui-même. L'exemple prouve que l'étude de la documentation archivistique doit être réalisée systématiquement sur tous les types de dossiers, même ceux qui, de prime abord, ne semblent pas utiles.

Il reste donc fort à faire, et la prochaine campagne portera notamment sur les archives de la Soprintendenza archeologica di Pompei. Il s'agira aussi d'élargir l'enquête à d'autres types d'archives. Par exemple, les témoignages des voyageurs et visiteurs du site peuvent receler des informations précieuses. On sait en effet que la ville antique a attiré de nombreux érudits et artistes européens ${ }^{18}$, qui ont pu laisser des mémoires, dessins, lettres relatifs à leur passage à Herculanum. Pour les identifier, il faudra dépouiller le registre de visites tenu entre 1806 et 1856 et qui est aujourd'hui conservé à l'Archivio storico de la Surintendance de Campanie à Naples. Par ailleurs, la consultation des archives du MANN liées aux travaux de restauration des fresques et des objets peut s'avérer éclairante si l'on souhaite préciser le devenir de certaines pièces entrées au Musée, comme par exemple le fameux tableau représentant Persée et Méduse, qui avait suscité l'intérêt du roi.

17 Une journée d'étude prévue le samedi 5 novembre 2016 à Toulouse permettra de faire le point sur l'apport des ressources archivistiques (textes mais surtout images - plans, dessins et photographies) et leur intégration dans le processus de production des reconstitutions infographiques. On comparera notamment le cas de la Casa d'Argo avec celui, mieux documenté et pour lequel la phase de recherche est plus avancée, de la Casa di Nettuno ed Anfitrite. 


\section{Recontextualiser les sculptures d'Herculanum}

L'étude des décors en contexte qui est au cœur du projet VESUVIA ne pouvait faire l'économie d'une enquête sur le décor statuaire, essentiellement en marbre et en bronze ${ }^{19}$ , issu des espaces domestiques d'Herculanum. Si le programme sculpté des édifices publics (théâtre, sanctuaires, "basilica » notamment) est beaucoup mieux connu et a davantage retenu l'attention des chercheurs que celui des demeures (à l'exception notable de la Villa des Papyri), la richesse et la variété des œuvres attestées dans les domus est néanmoins frappante. Des statuettes aux oscilla, des pinakes aux hermès, des décors de fontaine aux éléments de luminaire précieux, c'est une très vaste gamme d'objets d'art qui offre - du moins en première lecture - l'opportunité unique de saisir dans sa globalité l'univers figuratif dans lequel évoluaient au quotidien les habitants de la ville et de mieux comprendre comment dialoguaient images peintes et images sculptées ou ciselées. Dans ce contexte particulier, le terme générique de «décor statuaire » est particulièrement ambigu car nombre d'artefacts richement ornés avaient une finalité plus fonctionnelle que strictement décorative: il en va ainsi de plusieurs trapézophores marmoréens ou encore d'hermès à portraits exposés dans des atriums, qui pouvaient servir de support à un culte du genius du maître de maison ${ }^{20}$. Le cas des laraires est lui aussi tout à fait spécifique $^{21}$ : plusieurs d'entre eux accueillaient des statuettes d'une haute qualité d'exécution qui n'avaient pourtant pas de fonction ornementale première ${ }^{22}$. En revanche des œuvres telles que le relief votif des Nymphes et Charites, un original du début du III ${ }^{\mathrm{e}}$ s. av. J.-C. (fig. 8) provenant peut-être de l'île de Telos, ont le statut de véritables pièces de collection $^{23}$. Ces différences de fonctions et de statuts ${ }^{24}$ sont importantes lorsqu'il s'agit de dresser un inventaire recontextualisé des sculptures.

Fig. 8 - Relief des Nymphes, provenant d'Herculanum.

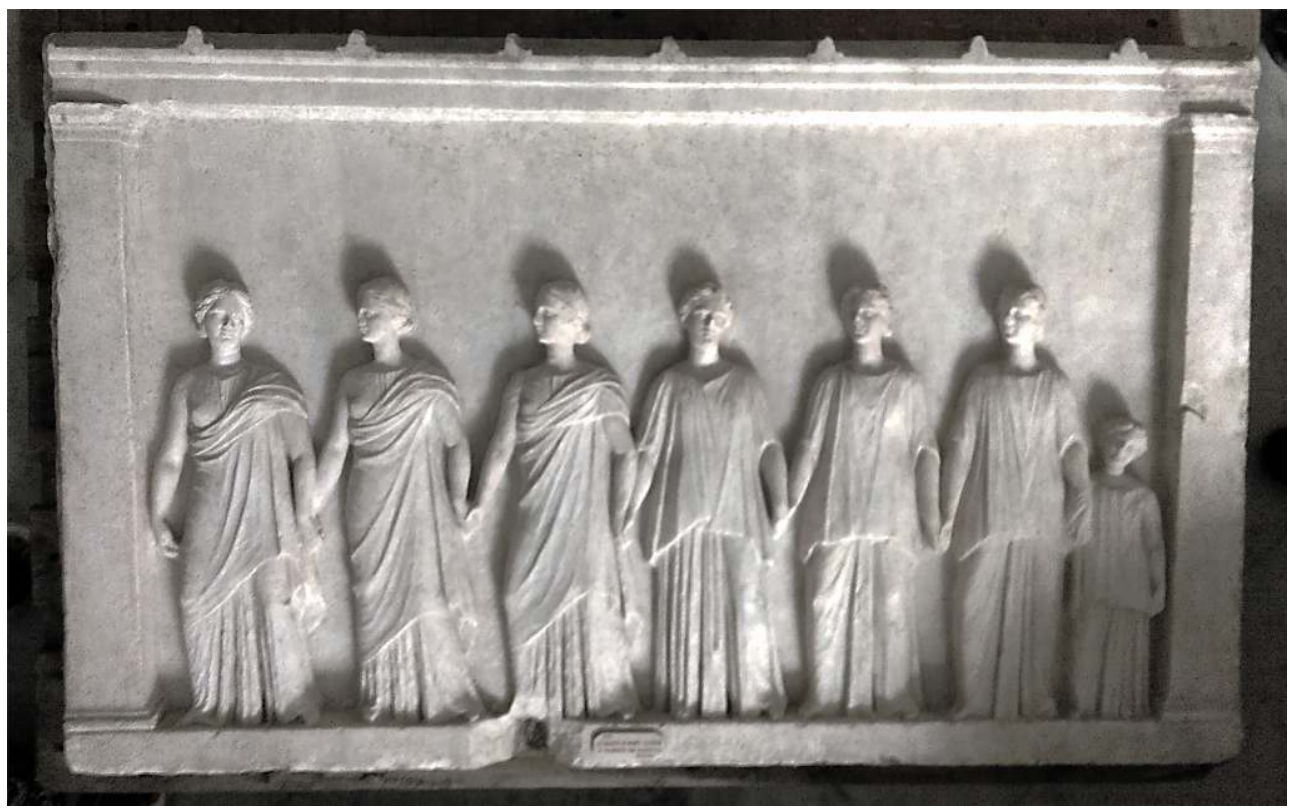

MANN, inv. n 6725 ; Cliché C. Marotta. Avec l'aimable autorisation de la Soprintendenza et du Ministero dei Beni e delle Attività Culturali e del Turismo. 
19 Pour comprendre le destin de ces œuvres depuis l'éruption de 79 apr. J.-C., il importe de rappeler que la statuaire relève d'un décor mobile, qui ne saurait par conséquent être traité de la même manière que les peintures pariétales, les stucs, le décor architectonique ou les pavements (mosaïques ou opus sectile). La nature même des objets a induit des différences dans leurs circonstances de découverte, leur transmission et leur conservation : les sculptures retrouvées in situ sont rarissimes à Herculanum. En effet, si les parois peintes ont souffert des effondrements ou des tunnels qui les ont irrémédiablement percées, les statues et les objets sculptés ont fréquemment été emportés de leur emplacement originel par le flux pyroclastique et parfois déplacés sur de grandes distances. En témoignent les objets arrachés aux étages supérieurs des bâtiments et découverts bien au-dessus du niveau de circulation antique, ou les fragments d'une même œuvre trouvés à plusieurs mètres de distance. Par conséquent, les lieux de découverte, même lorsqu'ils sont connus, ne peuvent être considérés comme des lieux d'exposition originels.

Plusieurs dizaines de statues ou statuettes issues des explorations du XVIII ${ }^{\mathrm{e}}$ siècle et conservées au MANN sont simplement inventoriées comme provenant d'Herculanum. Inversement, comment repérer aujourd'hui dans les réserves une œuvre dont le lieu de découverte est connu par les journaux de fouille mais qui se trouve simplement désignée comme «statuette de Minerve»? Les illustrations d'œuvres considérées comme «mineures" sont rarissimes et leur description extrêmement sommaire; seuls le croisement des données hétérogènes issues des publications anciennes, des inventaires successifs du Musée et des documents de fouilles (pour les découvertes les plus récentes) permettent dans certains cas de sortir de l'impasse : les associations d'objets, la présence de motifs rares ou particulièrement remarquables et les dates de mise au jour sont d'une aide précieuse. Les notices relatives aux restaurations des œuvres en marbre aident également à préciser certaines provenances.

21 Les premières missions de recherche consacrées aux objets statuaires ont été effectuées en parallèle au MANN, à l'Ufficio catalogo, à l'Ufficio fotografico et à l'Archivio storico de la Surintendance et ont porté plus spécifiquement sur les reliefs en marbre. Cette catégorie d'objets a été retenue en premier lieu en raison des conditions d'exposition particulières de certains d'entre eux, qui étaient fixés sur les parois des pièces qu'ils ornaient: ils constituent donc les rares objets sculptés susceptibles d'avoir été découverts à leur emplacement originel. En outre, du fait de leur insertion dans des parois peintes, qui constituent l'un des objets d'études privilégiés du projet VESUVIA, il nous a semblé que ces reliefs constituaient des témoignages importants de la complémentarité entre décor sculpté et peintures pariétales, les typoi de marbre pouvant se substituer dans certains cas aux tableaux peints. De fait, en 2009, a été mis au jour un relief mythologique, dont l'interprétation reste discutée ${ }^{25}$ (Herculanum, dépôt archéologique, inv. $n^{\circ} 88091$ ), qui était encore in situdans une pièce ornée de peintures appartenant à un espace de représentation d'un édifice résidentiel de l'insula I. Il était encastré à une hauteur de deux mètres au-dessus du sol, au même niveau qu'un autre relief trouvé en 1997 sur la paroi sud de la même pièce (Herculanum, dépôt archéologique, inv. $n^{\circ} 79613^{26}$ ) figurant deux satyres et une nymphe buvant du vin auprès d'une source. Le parement en réticulé avait été surcreusé pour recevoir les reliefs et leur fixation était assurée par de puissantes agrafes métalliques; l'enduit mural avait ensuite recouvert les bords des reliefs. Cet exemple particulièrement bien documenté, qui suggère l'existence d'un programme iconographique concerté dépassant le simple souci de créer une atmosphère dionysiaque 
${ }^{27}$, évoque directement par sa mise en scène celui du relief de Télèphe (MANN, inv. $\mathrm{n}^{\circ}$ 286787), lui aussi inséré dans une paroi de la pièce 17 de la maison du même nom. Il permet d'envisager des dispositifs comparables pour d'autres reliefs relevant de la même catégorie, qui sont relativement nombreux (une quinzaine au total au seul MANN). Or des trous d'agrafes du même type ont effectivement été repérés sur plusieurs d'entre eux, en particulier le célèbre relief polychrome du thiase bachique (fig. 9- MANN, inv. ${ }^{\circ} 6726$ ) ou encore le relief des Nymphes et Charites (MANN, inv. $n^{\circ} 6725$ ), ce qui confirme l'hypothèse d'une fixation à une paroi: pour ce dernier, une cassure visible à l'emplacement d'un goujon métallique pourrait témoigner des difficultés des «cavamonti » à arracher le relief au mur qui l'accueillait.

Fig. 9 - Relief figurant un cortège dionysiaque.

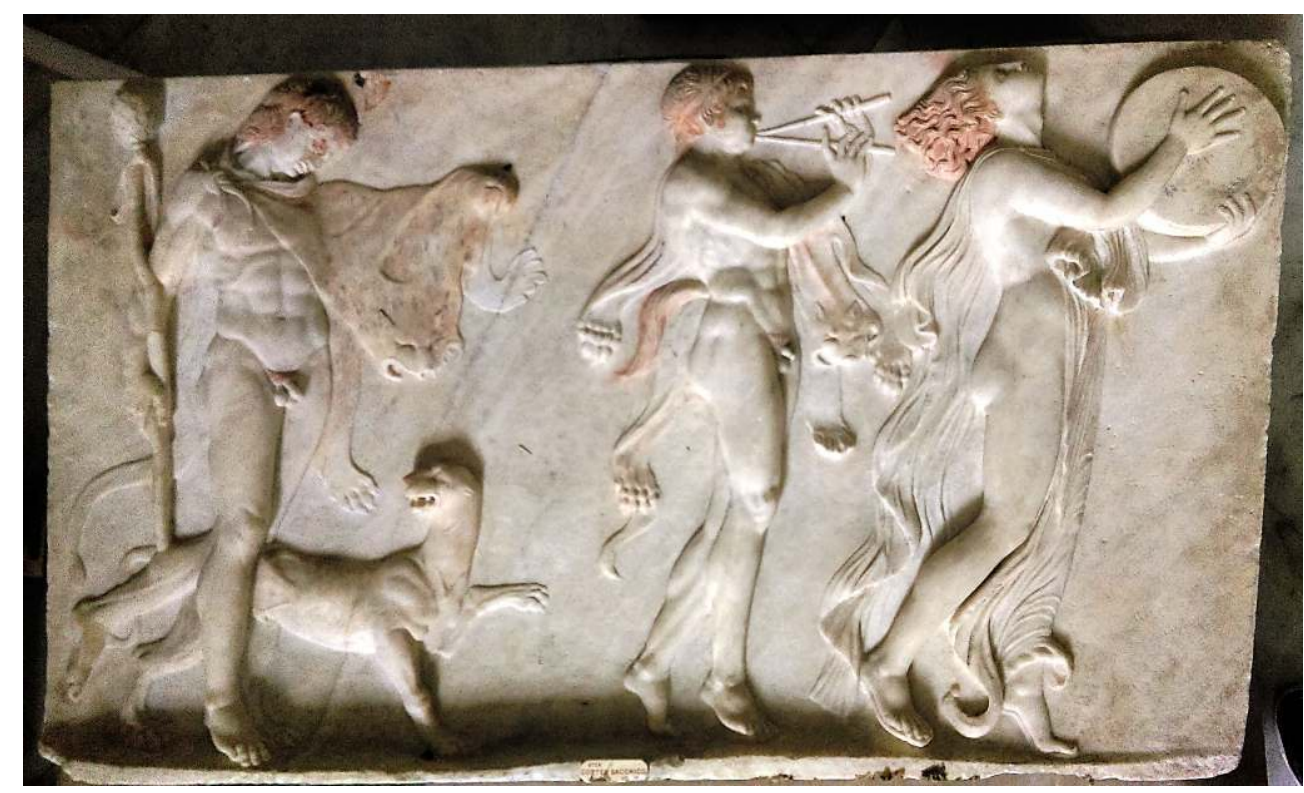

MANN inv. n6726; Cliché E. Rosso. Avec l'aimable autorisation de la Soprintendenza et du Ministero dei Beni e delle Attività Culturali e del Turismo.

Une poursuite des recherches en archives tentera de préciser leur lieu de découverte et leur étude technique et iconographique devra être complétée par celle des oscilla issues des fouilles d'A. Maiuri ${ }^{28}$; elle permettra de préciser à la fois leur datation et les modalités de leur mise en scène dans l'espace domestique de la cité vésuvienne.

\section{Avancée des restitutions 2D et 3D de la Casa di Nettuno ed Anfitrite}

La restitution infographique et la projection dans le modèle 3D réalisé par Archéovision des éléments de décor et de mobilier de la Casa di Nettuno ed Anfitrite sont l'objet d'un travail collectif particulièrement attentif (fig. 10- Axonométrie Archéovision). 
Fig. 10 - Axonométrie de la Casa di Nettuno ed Anfitrite.

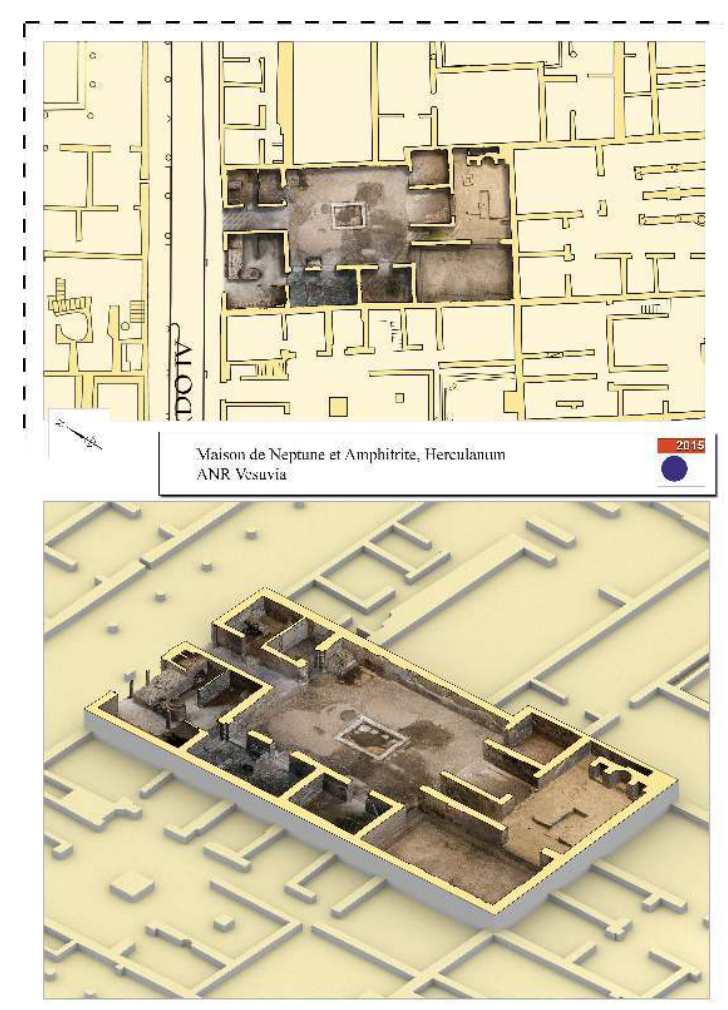

RÉALISATION : ARCHÉOVISION. AVEC L'AIMABLE AUTORISATION DE LA SOPRINTENDENZA ET DU MINISTERO DEI BENI E DELLE ATTIVITÀ CULTURALI E DEL TURISMO.

\section{Casa di Nettuno ed Anfitrite, restitution de la pièce 4}

Afin de compléter les restitutions graphiques de la Casa di Nettuno ed Anfitrite, l'étude de la pièce 4 a été entreprise. Ce petit cubiculum $(3,62 \times 2,45 \mathrm{~m})$ situé à gauche du tablinum ouvre à l'ouest directement sur l'atrium et à l'est, par une fenêtre haute, sur le nymphée.

La fouille de la pièce 4 , alors numérotée 10 , n'a laissé que peu de traces dans les archives. La seule entrée, datée du 9 janvier 1934 ne mentionne que la découverte d'un fragment d'amphore portant une inscription peinte en noir ${ }^{29}$. La description de la pièce, rédigée après l'achèvement des travaux de restauration et insérée à la fin du mois de juin 1934, détaille la décoration pariétale. Si l'interprétation qui en est donnée est discutable ${ }^{30}$, la description proprement dite est en revanche particulièrement précise.

Malgré les lacunes, les concrétions et l'usure, son état de conservation est meilleur que celui des pièces adjacentes puisque des éléments de ses quatre murs subsistent et qu'ils se répartissent sur toutes les zones des parois. Cela permet donc de repérer la structure décorative, de vérifier son identité sur les quatre parois, et de compléter les éléments lacunaires par ceux qui sont encore lisibles. Par ailleurs, il est possible de repositionner quelques prélèvements de l'époque des Bourbons (fig. 11). 
Fig. 11 - Casa di Nettuno ed Anfitrite, pièce 4 : restitution du décor.

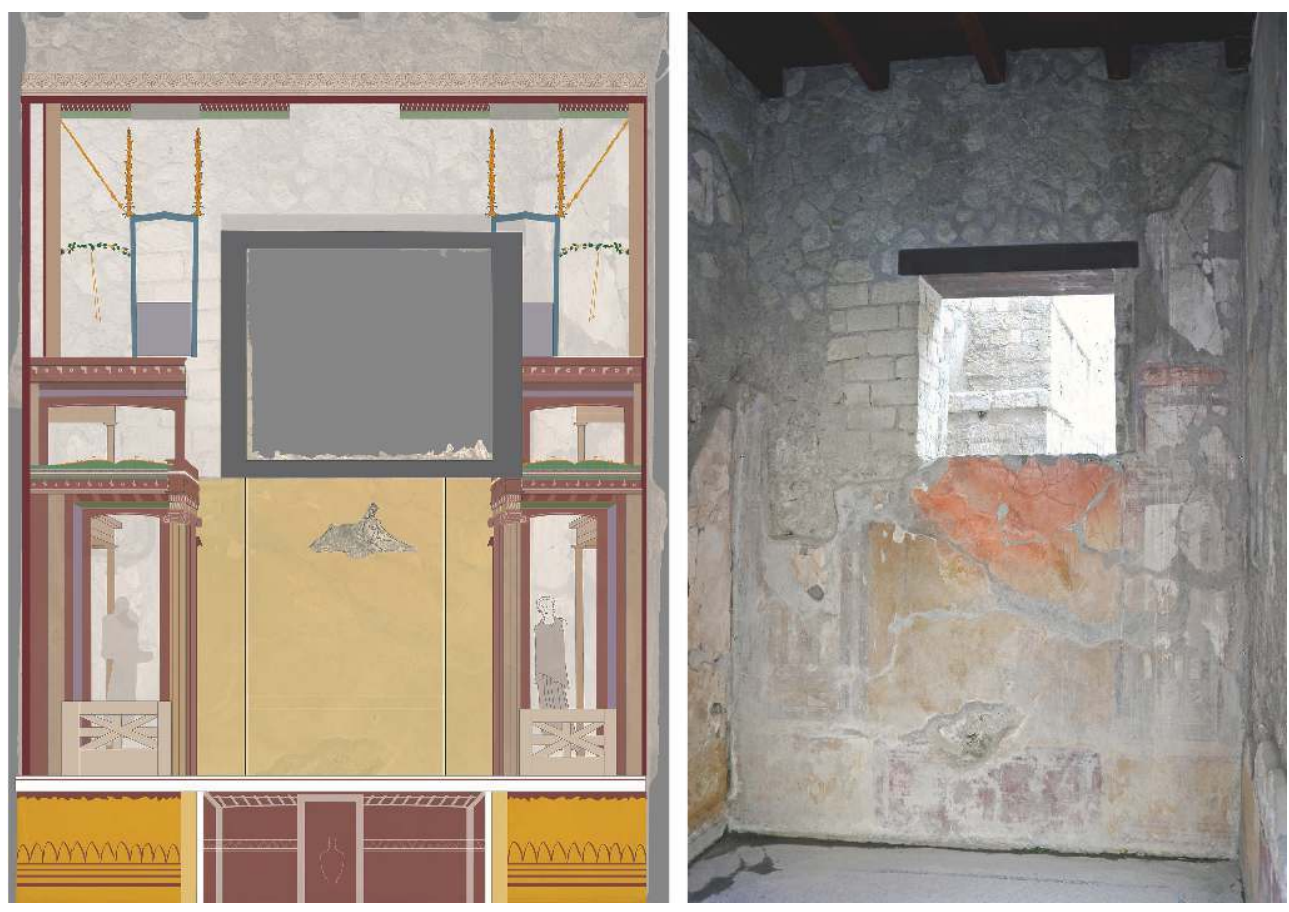

H. Eristov et M.-L. Maraval. Avec l'aimable autorisation de la Soprintendenza et du Ministero dei Beni e delle Attività Culturali e del Turismo.

En zone inférieure, l'enduit est presque intégralement conservé. On observe que sa structure est identique sur toutes les parois : un panneau central rouge bordeaux encadré de deux massifs piédestaux ocre jaune. En revanche le détail des motifs picturaux, très usé, concrétionné par endroits, manque de lisibilité et nécessite une lecture attentive. Il apparaît donc que le panneau rouge central a reçu un traitement architectural : il est couronné par un plafond à caissons reposant à l'arrière-plan sur deux supports, et son centre est occupé par un pseudo-édicule schématique enfermant, sur le mur sud, un haut vase cylindrique, et sur les autres murs un motif peu discernable. Sur les murs nord et sud, une fine guirlande relie cet édicule aux supports du plafond à caissons ; sur les murs est et ouest c'est une bordure ajourée horizontale. Le traitement des massifs piédestaux se restitue grâce aux sections les plus lisibles : leurs moulures inférieures se traduisent par une série d'horizontales plus ou moins larges, rouge-bordeaux; elles sont immédiatement surmontées par une rangée de hautes feuilles d'eau qui ornent environ un tiers de la hauteur du corps des piédestaux; la limite supérieure de ces massifs architecturaux, apparemment dépourvue de moulures, ne comporte pas non plus de corniche, mais la présence de celle-ci est suggérée par un large tracé rouge-bordeaux sinueux et irrégulier censé représenter l'ombre portée par la corniche. Vers le centre de chaque paroi, le petit côté fuyant de chaque piédestal semble n'avoir été rendu que par un ocre jaune plus clair que la face : aucune ligne de fuite n'a pu être repérée.

En zone médiane, la même structure tripartite se répète et ses divisions correspondent à celles de la zone inférieure sans toutefois que l'on observe un alignement parfait des axes verticaux. Un champ central ocre jaune est encadré de deux échappées architecturales qui prennent appui sur les piédestaux de la zone basse. Pour restituer l'aspect du champ central, on ne dispose que d'une donnée : les traces d'entablement en saillie sur les murs 
est et nord indiquent la présence d'un cadre architectural sous la forme d'un édicule, mais aucun élément de son couronnement ou du détail de son aspect ne subsiste. La restitution proposée reste donc parfaitement neutre. Une autre incertitude touche la présence de tableaux ou de figures au centre des murs nord et sud, très lacunaires, et sur le mur ouest à droite de la porte. Sur le mur oriental, en revanche, l'espace disponible sous la fenêtre a permis d'insérer une vignette : encore discernable dans les années 1970, à peu près disparue aujourd'hui, elle représente une figure féminine demi-couchée, tournée vers la droite, son coude droit appuyé sur un rocher, dans l'attitude d'une divinité fluviale ou d'une source. Cette hypothèse se trouve renforcée par le fait que ce mur est mitoyen de celui du nymphée et qu'il peut lui faire écho.

Les échappées de la zone médiane ne sont conservées que sur le mur est; mais dans la mesure où des lambeaux de leur couronnement subsistent sur le mur sud, il est très probable qu'elles se répétaient, identiques, sur tous les murs. À deux niveaux, elles affectent la forme d'un haut édicule à épi, couronné d'un fronton vert en accolade ; il est fermé par une barrière à croisillons derrière laquelle se tient une figure féminine debout sous un plafond à caissons, et débouche sur un espace ouvert où apparaît un portique fuyant. Le second niveau, peu développé, s'ouvre, au-delà d'une baie cintrée, sur une section de portique frontale. L'entablement qui surmonte l'ensemble de l'échappée est traité en alternance de bucranes très stylisés et de rosettes, identique, mais à une échelle légèrement supérieure, à celui qui surmonte l'édicule du premier niveau. L'épi qui flanque chacune des échappées en limite avec le champ central est constitué de deux colonnes ioniques cannelées sans base visible et portant un entablement fuyant.

La zone supérieure à fond blanc, également tripartite, s'organise de part et d'autre d'un édicule frontal à entablement plat, couvert d'un plafond à caissons et dont le fond semble bleu-gris : comme la seule attestation en est son lambeau gauche sur le mur sud, on ne peut affirmer s'il enfermait une figure ou un motif. Les zones latérales se subdivisent à leur tour ; le tiers central est constitué d'un pseudo-édicule à fronton linéaire en accolade abritant un griffon en vol; il se prolonge en un second niveau délimité par de fins candélabres végétaux et abritant un Amour en vol. Dans les tiers latéraux, une bordure ajourée horizontale sert de support à un perroquet (vers les angles) et un paon (vers l'édicule), sous une guirlande en arc.

31 Si cette structure se répète d'une paroi à l'autre, on note que le pseudo-édicule, vert sur les murs sud - et donc aussi nord -, est bleu sur les murs est - et donc aussi ouest.

Grâce à la présence d'un paon et d'un perroquet sur la partie gauche du mur sud, il a été possible de retrouver leurs pendants, prélevés au XVIII ${ }^{\mathrm{e}} \mathrm{s}$. : ce sont les deux fragments trouvés le 26 mars 1746 puis réunis sous un même numéro d'inventaire (MANN inv. $\mathrm{n}^{\circ}$ 8703), restitués en zone supérieure symétriquement à droite du mur sud. La bordure ajourée sur laquelle ils posent, complètement illisible in situ, a gardé intact son motif de postes qui a donc été rétabli graphiquement sur l'ensemble de la zone supérieure.

\section{Mise en œuvre et caractéristiques}

L'examen attentif du décor de cette pièce en vue de sa restitution amène à évaluer ses caractéristiques. En effet, il s'inscrit dans une parfaite cohérence avec ceux de l'atrium et du tablinum dont la zone basse se structure de la même façon (grands panneaux rouge bordeaux alternant avec des massifs architecturaux qui supportent les échappées de la zone médiane) et qui présentent de grands similitudes de mise en œuvre. Ces pièces ont 
donc fait l'objet d'une même campagne décorative et trahissent l'intervention d'un même atelier.

D'autre part, on constate que, de façon générale, ce décor qui vise à l'effet avec son partipris scénographique et l'aspect imposant de certains de ses éléments architecturaux, a pourtant été exécuté de façon assez peu soignée; de forts écarts dimensionnels (allant jusqu'à $10 \mathrm{~cm}$ dans une pièce de dimensions aussi réduites) se remarquent entre les zones symétriques d'un même mur ; les candélabres de la zone supérieure sont mal positionnés par rapport au sommet des pseudo-édicules censés les supporter; le rendu des faces fuyantes des gros piédestaux est déficient (même compte tenu des lacunes), et l'absence de corniche supérieure, simplement remplacée par son ombre portée, laisse à désirer. D'un point de vue technique, la peinture a été posée sur un enduit trop sec, ce qui a amené la perte complète des détails : les bordures ajourées, les entablements, les filets verticaux de la zone médiane du mur est, les motifs du socle, ont disparu pratiquement sans laisser de traces. En revanche, lorsque les fonds sont convenablement préparés, l'usure des motifs de détail laisse néanmoins dans la couleur de fond une empreinte repérable. En zone supérieure du mur sud, le pseudo-édicule vert clair qui abrite le griffon laisse transparaître des traits de pinceau préparatoires assez désordonnés.

L'impression générale que l'on retire de ce décor nuance donc l'opinion d'A. Maiuri qui voyait, dans cette maison, l'une des réalisations les plus fastueuses. Si l'occupant de la Casa di Nettuno ed Anfitrite avait, pour sa maison, de grandes prétentions à l'élégance, il n'en reste pas moins qu'il n'a pas dû en confier la réalisation aux meilleurs ateliers et qu'il a privilégié l'effet immédiat au détriment de la qualité.

\section{Casa di Nettuno ed Anfitrite, restitution du décor de l'atrium}

La fouille de l'atrium, alors numéroté 3, est mentionnée entre le $1^{\text {er }}$ mars et le 17 juin 1933. Tout au long de ce dégagement, les descriptions sont limitées à l'essentiel, lorsque des objets, inventoriés ou non, sont découverts, à l'exception de l'autel du culte domestique situé dans l'angle nord-ouest de la pièce. Indiqué comme s'étant trouvé sur le parcours d'un tunnel du XVIII ${ }^{\mathrm{e}}$., la découverte de cet autel témoigne d'une pratique assez rare. En effet, alors que des pièces de marbre peintes sont mises au jour à proximité, elles ne sont ni inventoriées sur le moment, ni particulièrement décrites, à l'exception de deux très rapides mentions, l'une au fil de la fouille, la seconde lors de la description finale ${ }^{31}$. Ce quasi-silence contraste fortement avec l'enthousiasme d'A. Maiuri qui y voit de possibles pinakes monochromes ${ }^{32}$. Le dépôt de ces plaques peintes évoque l'idée d'une décoration pariétale inachevée, ce qui apparait confirmé par la mention rapide faite au détour de la lecture de l'inscription purpura (CIL IV, 10 480), indiquée comme peinte au-dessus d'un cadre à sujet mythologique disposé dans la partie supérieure de la paroi occidentale. Cette inscription ainsi laisse supposer que la décoration était en voie d'achèvement, seule la bordure des cadres (insérés après la réalisation du reste de la paroi ?) devant encore être mise en couleur.

Sans nous attarder sur les différents objets découverts dans cette pièce, dont l'inventaire détaillé est reproduit en annexe, il convient de souligner la mention, sans numéro d'inventaire, d'une gargouille de compluvium prenant la forme d'une tête de chien en terre cuite surpeinte de blanc, qui ne serait pas sans rappeler celles de l'atrium de la Casa sannitica. 

decorazione d'atrio (...) dovuta ad un artista di grande ricchezza coloristica, di sicura bravura, di ardimento e di fantasia inventiva, di un artista, infine specializzatosi, potremmo anche dire, nel genere della pittura architetturale barocca, e non alieno, come accade invece nei correnti decoratori della casa ercolanese, dall'inserire soggetti figurati e figure isolate e motivi animalistici nel campo della decorazione architettonica. $»^{33}$ L'enthousiasme de cette description ne peut que faire regretter non seulement sa quasidisparition, mais encore l'absence de documentation graphique ou photographique. Dans son état actuel, il ne reste que des lambeaux de décor, la plupart situés en zone basse, quelques-uns en zone médiane sur les murs nord et sud. En ce qui concerne la zone supérieure il ne subsiste qu'un fragment sur le tiers droit du mur est; sur le cliché des années 1930 publié par A. Maiuri une grande plaque d'enduit adhérait encore sur le mur est, au-dessus de la porte de la pièce $4^{34}$; elle a aujourd'hui disparu sans qu'un relevé ou une photographie de détail en ait été pris ; sur le même mur, une autre plaque surmontait les portes donnant sur l'andron et sur le triclinium 7: seul un lambeau est encore partiellement lisible ; la même photographie montre, à l'extrémité gauche du mur sud, un auvent qui protège une grande plaque en zone haute, disparue elle aussi.

L'enquête a donc consisté à tirer parti des éléments encore visibles en zone basse pour restituer le rythme des panneaux et inter-panneaux, et à exploiter les faibles traces de zone médiane (fig. 12).

Fig. 12 - Casa di Nettuno ed Anfitrite, atrium, mur nord.
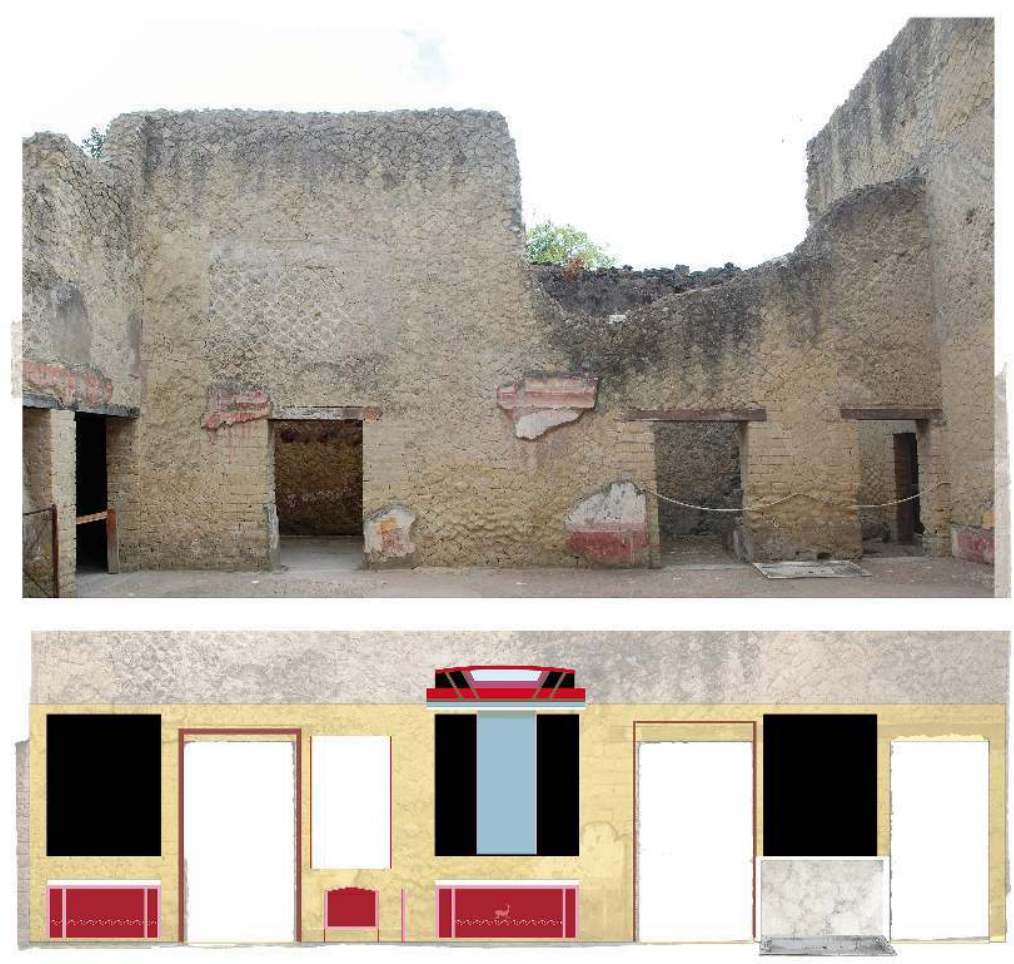

Proposition de restitution à partir des éléments in situ sur les murs nord et sud.

H. Eristov et M.-L. Maraval. Avec l'aimable autorisation de la Soprintendenza et du Ministero dei Beni e delle Attività Culturali e del Turismo. 

pratiquement pas de solution de continuité dans les enduits de sa zone basse. Malgré l'érosion de la couche picturale, il a donc été possible de relever les mesures des panneaux et des compartiments, et de les restituer sur l'orthophotographie. La partition décorative fait alterner de grands panneaux rouge bordeaux et des panneaux ocre jaune plus étroits, eux-mêmes animés par un compartiment rouge bordeaux, à couronnement cintré aux extrémités est et ouest de la paroi, et en bâtière pour les deux éléments centraux. Les grands panneaux rouges sont recoupés par des filets horizontaux et verticaux forment encadrement au centre duquel une ligne de sol porte un animal ou un monstre: l'un d'eux, apparemment un capriné tourné vers la gauche, est encore partiellement visible sur le panneau central, tandis que sur le panneau gauche, un quadrupède ailé à queue recourbée est tourné vers la droite. Les compartiments jaunes sont à comprendre comme de gros piédestaux dans lesquels s'inscrit une table de couleur contrastée; seul celui de l'extrémité est a conservé son motif: un vase en forme de cratère. Pour A. Maiuri, ce soubassement évoque le proscenium d'un pulpitum de théâtre.

41 La restitution de la zone médiane s'appuie d'une part sur ce rythme, d'autre part sur les vestiges faiblement lisibles à l'extrémité gauche du mur nord, enfin sur la plaque repositionnée sur le mur sud. S'y ajoute la notation d'A. Maiuri selon lequel les champs médians étaient constitués de grands édicules s'ouvrant entre de fastueuses perspectives architecturales. On peut donc établir que les "piédestaux » ocre jaune portaient des architectures dont on lit encore la succession de colonnes ou de supports ocre jaune, rose, violacé, gris, sans que l'on puisse en dire davantage sur leur organisation spatiale. Entre ces échappées et les panneaux noirs alignés sur la largeur des panneaux rouge bordeaux $\mathrm{du}$ soubassement, un large encadrement ocre jaune formait transition. C'est la plaque repositionnée au centre du mur sud qui fournit quelques données sur le couronnement de ces panneaux : malgré son effacement, on y lit encore les traces d'un plafond à solives en perspective ménageant un opaion central à fond clair, sans doute bleu. Le couronnement est cintré, et l'angle supérieur droit d'un tableau subsiste: par symétrie, on peut en restituer la largeur $(0,54 \mathrm{~m})$. Comme cela a déjà été noté pour le triclinium 7 , le positionnement de cette plaque lors des fouilles des années 1930 est légèrement fautif, et elle doit être décalée de $0,20 \mathrm{~m}$ vers la gauche. Cet édicule encadrant un tableau se trouvant au centre du mur sud, il est certain que son pendant sur le mur nord revêtait le même aspect ; quant aux édicules latéraux, ils devaient avoir un traitement voisin, sinon tout-à-fait identique. Ils étaient au nombre de deux sur le mur nord, probablement un seul sur le tiers gauche du mur sud (interrompu par trois portes), un sur le tiers gauche du mur ouest et un, sans doute un peu plus étroit, sur le tiers droit du mur est, au-dessus du laraire ; le mur est, interrompu par quatre portes, ne laissait aucun espace pour un panneau architectural, et, le soubassement ayant disparu, rien ne permet de proposer des hypothèses.

42 Dans l'état actuel de la documentation disponible, il n'a pas encore été possible de suggérer une restitution de la zone supérieure; cependant, l'analyse d'un cliché des années 1930 (Alinari 43124) fournira peut-être des éléments.

La structure décorative de l'atrium confirme le jugement d'A. Maiuri: l'important dispositif architectural constitué par de larges échappées à fond clair encadrant les panneaux bidimensionnels est moins courant dans les atria que dans d'autres pièces publiques ou de réception (tablinum, oecus, triclinium); en effet, la hauteur considérable des parois et leur surface discontinue irrégulièrement interrompue par des portes, se

Chronique des activités archéologiques de l'École française de Rome , Les cités vésuviennes | 2016 
prêtent mal aux effets «scénographiques ». Citons, cependant le vaste atrium 4 de la maison VIII 2, 18-21 à Pompéi, aujourd'hui perdu mais documenté par des photographies et par une planche d'A. Niccolini ${ }^{35}$ : comme dans celui de la Casa di Nettuno ed Anfitrite, l'un des murs (nord) disposait d'une surface continue. Le choix de ce système décoratif ambitieux signale donc, de la part du commanditaire, une volonté d'effet.

Cette volonté se manifeste également par l'abondance de panneaux noirs. Or on sait que ce choix chromatique implique une mise en œuvre complexe : le noir de carbone étant incompatible avec la technique de la fresque, il faut colorer l'enduit, puis poser une première couche, généralement rouge, enfin étendre le pigment noir mélangé à de la colle animale ${ }^{36}$. C'est sur le site d'Herculanum que les commanditaires ont le plus apprécié les murs noirs, ceci dans la cohérence de leur goût pour les matériaux rares et précieux ( opus sectile) et les fonds colorés coûteux (murs bleus) ou de réalisation complexe (murs verts).

La question qui se pose concerne la présence de tableaux dans l'atrium. Si l'enquête archivistique n'a pour l'instant permis de repérer aucun prélèvement de l'époque des Bourbons, en revanche, la plaque au centre du mur sud atteste très vraisemblablement la présence d'un tableau à fond bleu-verdâtre, et donc au moins d'un pendant sur le mur sud. Le journal de fouilles du $1^{\mathrm{er}}$ mars 1933 indique la présence, sur le mur ouest, d'un tableau mythologique non décrit au-dessus duquel se trouve l'inscription PVRPVRA. La mention de "soggetti figurati e figure isolate e motivi animalistici nel campo della decorazione architettonica $»^{37}$ sur les murs sud et est laisse supposer, soit que des figures en vol ou flottantes occupaient certains des édicules, soit que des figures occupaient les échappées architecturales en même temps que des animaux, des monstres ou des oiseaux, comme c'est le cas dans la pièce 4 . La difficulté d'identifier dans les collections du MANN de possibles éléments appartenant à l'atrium vient de ce que non seulement les journaux de fouille ne signalent rien, mais encore que nous ne disposons d'aucun décor in situ à partir duquel construire le raisonnement. Seul peut être restitué le schéma décoratif, ce qui, compte tenu de l'état avancé de dégradation de la pièce, constitue un apport intéressant.

\section{Les marbres peints signés d'Alexandros Athenaios : le problème de leur provenance ${ }^{38}$}

Dans les journaux de fouilles de la Casa di Nettuno ed Anfitrite, à la journée du 20 avril 1933 est mentionnée la découverte de plaques marbres peintes de sujets figurés, près du laraire 39. Ces reliefs furent portés directement au Musée National de Naples où ils furent inventoriés sous les numéros 150210 et $15211^{40}$. Il s'agit de fragments de deux plaques peintes, dont une porte la signature en grec d'un certain Alexandros Athenaios (MANN, inv. $n^{\circ} 15211$ ), déjà connue grâce à une autre plaque de marbre peint, trouvée lors des fouilles du XVIII ${ }^{\mathrm{e}}$ s. (MANN, inv. n 9 562). A. Maiuri, dans son ouvrage de 1958, décrit la découverte des fragments avec enthousiasme et mentionne aussi la plaque déjà connue avec la signature d'Alexandros Athenaios. Il s'agit de la représentation des «Joueuses d'osselets ${ }^{41}$ (fig. 13) Nous connaissons la date de mise au jour de cette œuvre : le 24 mai 1746. L'information se trouve dans le journal de J. Alcubierre, ainsi que dans les Antichità di Ercolano, la publication officielle des découvertes herculanéennes, où la peinture figurant les « Joueuses d'osselets » figure dans le premier volume, sur la planche I ${ }^{42}$. 
Fig.13 - Plaque de marbre peinte figurant les « Joueuses d'osselet ».

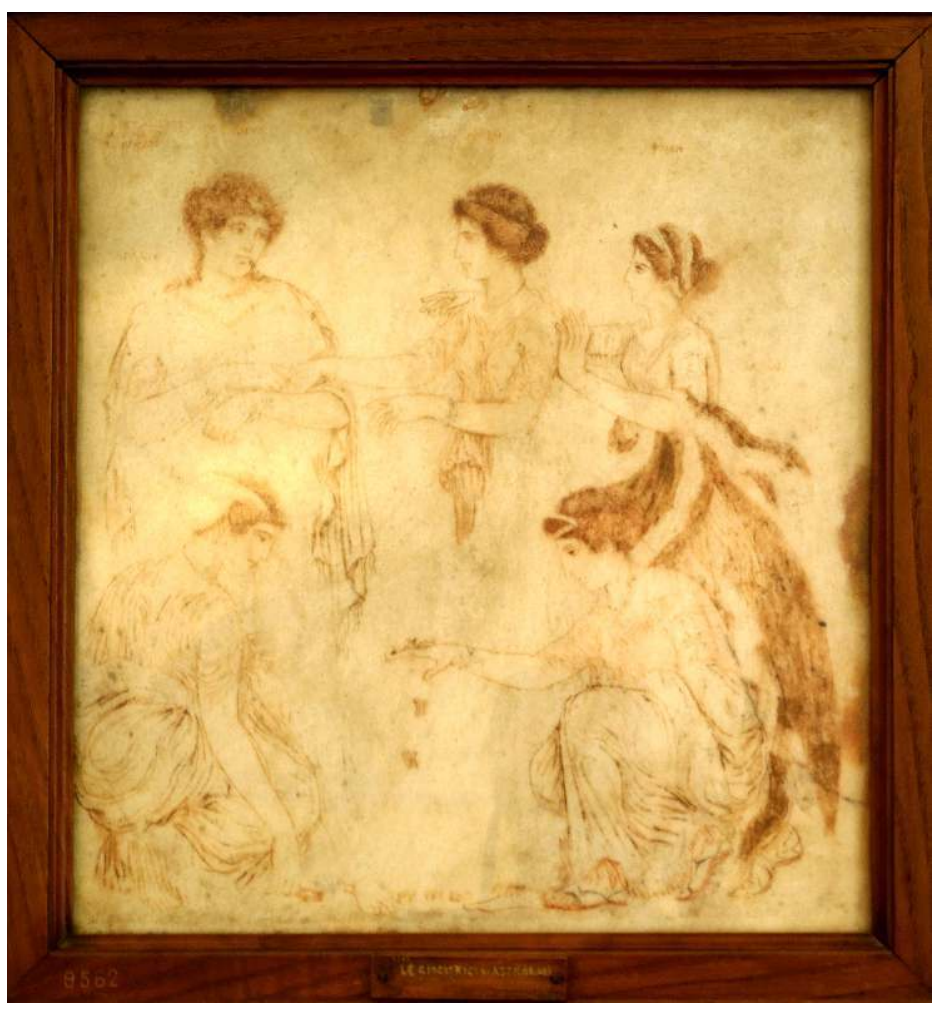

MANN, inv. nº 9562.

Nous connaissons donc deux plaques de marbre peintes et signées par Alexandros Athenaios, trouvées à une distance de presque deux siècles. La provenance est assurée pour les fragments mis au jour par A. Maiuri, tandis que nous ne connaissons que la date de découverte pour l'autre plaque. A. Maiuri ne parle pas du problème de la provenance de la plaque trouvée au XVIII ${ }^{\mathrm{e}}$ s., et $\mathrm{M}$. de Vos est la première à avancer l'hypothèse que la plaque des "Joueuses d'osselets" pourrait provenir de la Casa di Nettuno ed Anfitrite ${ }^{43}$. Dans le catalogue Ercolano. Tre secoli di scoperte, le rattachement des marbres peints découverts en 1746 à cette maison est présenté comme un fait ${ }^{44}$.

La présence des fouilleurs du XVIII ${ }^{\mathrm{e}}$ s. dans la Casa di Nettuno ed Anfitrite est documentée pour mars 1746. Le dernier prélèvement de peinture murale dans le triclinium de cette demeure advient le 21 mars 1746. L'identification des peintures prélevées dans la pièce 4 avec celles mentionnées à la date du 26 mars 1746 (voir plus haut) offre ici une autre date liée à la maison. Durant les deux mois entre l'ultime prélèvement documenté dans la Casa di Nettuno ed Anfitrite (26 mars 1746) et la date de découverte du marbre des "Joueuses d'osselets » (14 mai 1746), les fouilleurs travaillent alors dans l'édifice connu sous le nom de pistrinum (Ins. Or. II, 1a), où ils détacheront entre le 26 avril et le 9 mai 1746 une grande quantité de peintures de III $^{\mathrm{e}}$ style ${ }^{45}$. Fin avril 1746, sont mentionnés deux fragments de statue équestre qui laissent supposer qu'une équipe œuvre dans la zone où étaient les statues équestres de M. Nonius Balbus, trouvées le 10 juin et le 11 août 1746. Parmi les découvertes décrites dans le rapport du 24 mai 1746, outre la plaque peinte, sont mentionnés des fragments de statues ${ }^{46}$.

Dans une lettre du 17 mai 1746, J. Alcubierre parle de trois secteurs alors excavés : «... hallandose el travajo distribuydo con buen orden en 3. parajes, que son el lugar en que 
estava parte de la estatua á cavallo ; las Vecinidadas de los Edificios en que se encontraron las ultimas pinturas ; Y un otro lugar que como en los precedentes sempre se descubren otras habitaciones " ${ }^{47}$. Le lieu où se trouvaient des éléments de la statue équestre doit être identifié comme la place monumentale près du théâtre ${ }^{48}$, tandis que la maison à peintures est l'édifice décoré d'enduits $\mathrm{III}^{\mathrm{e}}$ style situé dans l'Ins. Or. II, 2; en revanche, nous ne savons pas quels autres bâtiments furent fouillés durant cette période. En définitive, quels sont les seuls éléments dont nous soyons certains? À Herculanum existaient plusieurs plaques de marbre peint. Deux d'entre elles, parmi celles découvertes, étaient signées d'Alexandros Athenaios, dont une appartenait au décor de la Casa di Nettuno ed Anfitrite. Quant au lieu de provenance de la fameuse plaque peinte des "Joueuses d'osselet », le mystère demeure.

Afin de communiquer de manière efficace et régulière sur les résultats du programme ANR VESUVIA, un carnet de recherches numérique a été ouvert sur le site sur hypotheses.org: http://vesuvia.hypotheses.org/ Les infographies et restitutions 3D y sont ainsi présentées, au fur et à mesure de leur réalisation. Ce carnet permet également d'annoncer les publications et événements scientifiques organisés dans le cadre du programme.

\section{BIBLIOGRAPHIE}

Adamo Muscettola 1984 = S. Adamo Muscettola, Osservazione sulla composizione dei larari con statuette in bronzo di Pompei ed Ercolano, dans U. Gehrig (éd.), Toreutik und figürliche Bronzen römischer Zeit, Berlin, 1984, p. 9-32.

Allroggen-Bedel 1974 = A. Allroggen-Bedel, Maskendarstellungen in der römisch-kampanischen Wandmalerei, Munich, 1974.

Allroggen-Bedel 1983 = A. Allroggen-Bedel, Dokumente des 18 Jahrhunderts zur Topographie von Herculaneum, dans Cronache Ercolanesi, 13, 1983.

Allroggen-Bedel 2009 = A. Allroggen-Bedel, Ercolano, dal sito al museo, dal museo agli archivi : il puzzle ercolanese, dans A. Coralini (éd.), Vesuviana : archeologie a confronto [Atti del Convegno internazionale (Bologna, 14-16 gennaio 2008)], Bologne, 2009, p. 167-179.

Allroggen-Bedel $2010=$ A. Allroggen-Bedel, A proposito dei Balbi : note archivistiche alla topografia d'Ercolano, dans C. Gasparri, G. Greco, R. Pierobon Benoit (éd.), Dall'immagine alla storia : studi per ricordare Stefania Adamo Muscettola, Naples, 2010 (Quaderni del Centro Studi Magna Grecia, 10), p. 355-373.

Antichità di Ercolano 1757 = Le Antichità di Ercolano esposte con qualche spiegazione, vol. I, Naples, 1757.

Antoni 2009 = A. Antoni, Voyageurs français à la découverte d'Herculanum aux XVIII ${ }^{\text {ème }}$ et XIX ${ }^{\text {ème }}$ siècles, dans Cronache Ercolanesi, 39, 2009, p. 313-330.

Böhm 2008 = S. Böhm, Labung an der Wein-quelle : ein dionysisches Marmorrelief in Herculaneum, dans JdI, 123, 2008, p. 171-197. 
Bragantini - Sampaolo 2009 = I. Bragantini, V. Sampaolo, La Pittura Pompeiana, Milan, 2009. Budetta - Pagano 1988 = T. Budetta, M. Pagano, Ercolano, legni e piccoli bronzi : testimonianze dell'arredo e delle suppellettili della casa romana, Rome, 1988 (Mostre della Soprintendenza archeologica di Pompei, 3).

Cadario 2015 = M. Cadario, Le statuette in bronzo in contesto tra culti, ornamenta e pezzi da collezione, dans B. Arbeid, M. Iozzo (éd.), Piccoli grandi bronzi : capolavori greci, etruschi e romani delle collezioni mediceo-lorenesi nel Museo archeologico nazionale di Firenze, Florence, 2015, p. 53-59.

Caruso 2011 = F. Caruso, Melampo e le figlie di Preto : una lettura del nuovo rilievo di Ercolano, dans RStPomp, 22, 2011, p. 25-35.

Cinquantaquattro - Capaldi - Sampaolo 2014 = T.E. Cinquantaquattro, C. Capaldi, V. Sampaolo (éd.), Augusto e la Campania : da Ottaviano a Divo Augusto, 14-2014 d.C., Milan, 2014.

Ciotola 2013 = A. Ciotola, Ancora sul rilievo neoattico di Ercolano $:$ una diversa lettura, dans RStPomp, 24, 2013, p. 33-40.

Dardenay et al. 2015 = A. Dardenay, A. Allroggen-Bedel, H. Eristov, M.-L. Maraval, N. Monteix, Habitat et société à Herculanum, dans Chronique des activités archéologiques de l'École française de Rome, 2015 [en ligne] (http://cefr.revues.org/1339).

De Vos - De Vos 1982 = A. De Vos, M. De Vos, Pompei Ercolano Stabia, Rome-Bari, 1982.

Finati 1839 = G. Finati, Perseo assistito da Minerva che tronca il capo a Medusa, dans Real museo Borbonico, XII, 1839, p. 1-10.

Guidobaldi 2008 = M. P. Guidobaldi (ed.), Ercolano. Tre secoli di scoperte, Naples, 2008.

Herbig 1962 = R. Herbig, Nugae Pompeianorum, Tübingen, 1962.

Kaufmann-Heinimann 1998 = A. Kaufmann-Heinimann, Götter und Lararien aus Augusta Raurica : Herstellung, Fundzusammenhänge und sakrale Funktion figürlicher Bronzen in einer römischen Stadt, Bâle, 1998 (Forschungen in Augst, 26).

Maiuri 1958 = A. Maiuri, Ercolano. I nuovi scavi, 1927-1958, Rome, 1958.

Moormann 1986 = E. M. Moormann, Un fior di giardino ed altri frammenti di pittura ercolanesi, dans Cronache Ercolanesi, 16, 1986, p. 123-133.

Monteix 2010 = N. Monteix, Les lieux de métier : boutiques et ateliers d'Herculanum, Rome, 2010 ( BEFAR, 344).

Niccolini 1896 = A. Niccolini, Le case ed i monumenti di Pompei disegnati, vol. IV, Naples, 1896.

Pagano 2005 = M. Pagano, I primi anni degli scavi di Ercolano, Pompei e Stabiae : raccolta e studio di documenti e disegni inediti, Rome, 2005 (Studi della Soprintendenza archeologica di Pompei, 11).

Pagano - Prisciandaro 2006 = M. Pagano, R. Prisciandaro, Studio sulle provenienze degli oggetti rinvenuti negli scavi borbonici del Regno di Napoli, Castellammare di Stabia, 2006.

Pannuti 1983 = U. Pannuti, Il « giornale degli scavi » di Ercolano (1738-1756), dans Atti della Accademia nazionale dei Lincei, Memorie della Classe di scienze morali, storiche e filologiche, 26, 1983, p. 163-410.

Ruggiero 1885 = M. Ruggiero, Storia degli scavi di Ercolano ricomposta su' documenti superstiti, Naples, 1885.

Schefold 1957 = K. Schefold, Die Wände Pompejis, Berlin 1957. 


\section{ANNEXES \\ JOURNAUX DE FOUILLE, INVENTAIRES ET CARNETS DE M. DELLA CORTE CONCERNANT LA FOUILLE DE L'ATRIUM ET DE LA PIÈCE 4}

Note : dans les inventaires, le texte principal est celui reporté dans les GSE, les éventuels ajouts ou modifications provenant des livres d'inventaire sont reportés entre accolades $\{$. Le texte reporté entre parenthèses () correspond aux mentions supplémentaires dans les GSE par rapport aux inventaires. Pour les objets non inventoriés mais mentionnés parce qu'ils présentent une inscription, après la description reportée dans les GSE sont reproduites les notes prises par M. Della Corte dans ses carnets conservés au Getty Research Institute. Le texte des inscriptions reporté au milieu des paragraphes des GSE correspond à celui du Corpus.

\section{Atrium}

1933

1 marzo. [...] Nella casa $\mathrm{N}^{\circ} 6$ sul IV cardine, lato est, nella parete ovest sopra un riquadro di un soggetto mitologico vi è la seguente iscrizione fatta con pittura : purpura ${ }^{49}$.

È lunga m. 0.085 e le lettere sono alte m. 0.03 .

8 marzo. Nell'ambiente n. 3 [della casa $\mathrm{N}^{\circ} 6$ sul $4^{\circ}$ cardine, lato est (07/03/1933)], sul pavimento, a m. 1.20 dalla parete sud ed a m. 0.80 da quella ovest è stata raccolta : [Description des objets inventoriés 1040 à 1043].

11 marzo. Nella casa $\mathrm{N}^{\circ} 6$ sul $4^{\circ}$ cardine, lato est, ambiente n. 3, a m. 0.30 dal pilastro del vano che mena nell'altro ambiente n. 4, a m. 2.30 dal vano della parete sud ed a $0.40 \mathrm{di}$ altezza dal pavimento è stato raccolto : [Description des objets inventoriés 1053 à 1055].

7 aprile. Si lavora nella casa $\mathrm{N}^{\circ} 6$ sul $4^{\circ}$ cardine lato est. È stato raccolto sul pavimento dell'ambiente n. 3 una testa di cane di terracotta policromata di bianco. Essa fa parte della decorazione del tetto del compluvio.

19 aprile. Nella casa $\mathrm{N}^{\circ} 6$ sul $4^{\circ}$ cardine, lato est, ambiente n. 3, nell'angolo nord est [ovest] vi è la parte bassa di un'ara votiva di marmo, la parte alta fu asportata da un cunicolo borbonico. Aderente alla parete est, in un fosso prodotto dal peso del materiale è stato raccolto : [Description des objets inventoriés 1059 et 1060].

20 aprile. Si è ripigliato il lavoro sul vuoto che trovasi nell'ambiente 3 , della casa $\mathrm{N}^{\circ} 6$, sul $4^{\circ}$ cardine, lato est. Molti frammenti di marmi colorati sono stati raccolti, marmi che potevano adornare l'ara votiva ieri accennata. Due lastre di marmo una della lunghezza di m. 0.39 e l'altra m. 0.50, l'altezza manca perché la parte superiore è spezzata, si sono anche raccolte e sopra esse vi sono delle figure fatte con colori vari. Si è anche raccolto : [Description des objets inventoriés 1061 à 1063].

22 aprile. [...] Nella casa $\mathrm{N}^{\circ} 6$ sul $4^{\circ}$ cardine si lavora nell'ambiente n. 3. Nessun trovamento.

24 aprile. [...] Nella casa $\mathrm{N}^{\circ} 6$ nello stesso cardine, lato est, nell'atrio è stato raccolto : [Description des objets inventoriés 1072 et 1073]. 
26 aprile. Nell'atrio della casa $\mathrm{N}^{\circ} 6$ sul $4^{\circ}$ cardine, lato est, sul pavimento è stato raccolto : [Description d'un bord de vase sigillé].

2 maggio. Nell'ambiente $\mathrm{N}^{\circ} 3$ della Casa 6, IV Cardine, lato Est è stata raccolta :

[Description d'un col d'amphore pourvu d'inscriptions peintes].

9 maggio. Nella Casa n. 6 , sul IV Cardine, lato Est, tra la parete e l'Ara votiva che trovasi nell'angolo Nord-ovest [de l'atrium 3], vi erano i seguenti oggetti : [Description des objets inventoriés 1081 à 1084].

15 maggio. [...] Nell'atrio della stessa Casa [n. 6 posta sul IV Cardine, lato est] è stato raccolto : [Description d'un bord de vase sigillé].

6 giugno. Nella Casa n. 6 , sullo stesso Cardine, è stato sterrato il trave principale del compluvio. Misura m. 0.45 di altezza per m. 0.18 di spessore.

16 giugno. Nell'atrio della Casa n. 6 , IV Cardine, lato Est è stato raccolto : [Description de l'objet inventorié 1096].

Nello stesso ambiente si è sterrato : [Description d'un fragment de tuile marquée].

17 giugno. Nella Casa n. 6, sul IV Cardine, lato Est, nell'Ambiente $\mathrm{N}^{\circ} 3$, atrio, sul pavimento a m. 0.30 della vasca dell'impluvio, lato Nord, è stato sterrato : [Description de l'objet inventorié 1097].

Description générale de l'atrium (ambiente 3) : È l'atrio tuscanico con cisterna e ara votiva nell'angolo nord ovest. Misura m. 9. 05 di lunghezza per m. 7.20 di larghezza. Intorno ad esso vi sono 9 vani distribuiti 3 a sud ; 4 est e due ad ovest. L'impluvio è di marmo bianco e misura m. 1.54 di larghezza per m. 2.05 di lunghezza. Il bordo è alto m. 0.20 per 0.25 ed il fondo è di lastre di marmo. Sul fondo dell'impluvio vi sono quattro buchi, uno quello sulla parete est, serviva per il discarico dell'acqua dal triclinio estivo, quello sulla parete ovest faceva calare l'acqua nella cisterna e gli altri immettevano l'acqua di una fontana. La bocca della cisterna è del diametro di m. 0.64. La profondità non è conosciuta perché non è stato spurgato. I travi del compluvio sono di forma rettangolare e misurano m. $0.30 \mathrm{di}$ altezza per m. 0.20 di spessore e sono posti da nord a sud. I travicelli che sostenevano il tetto misuravano m. 0.10 per $0.9 \mathrm{e} \mathrm{la} \mathrm{distanza} \mathrm{che} \mathrm{passa} \mathrm{tra} \mathrm{l'uno} \mathrm{e} \mathrm{l'altro} \mathrm{è} \mathrm{di} \mathrm{m.} \mathrm{0.31.} \mathrm{Essi}$ sono a forma rettangolare. L'ara votiva che trovasi nell'angolo nord ovest, solamente la base esiste è di $\mathrm{m} .1$ di lunghezza per m. 0.84 di larghezza. Era rivestita di marmo bianco. Nei pressi dell'ara furono raccolti i due marmi dipinti e la statua di Ercole nudo. Il pavimento è di signino con filari di frammenti di marmi di dimensioni e colori differenti. La parete nord, la parte alta e centrale, conserva poca decorazione, mentre lo zoccolo vi è per intero. Esso è di stucco a fondo rosso con fasce di giallo. Sul fondo rosso vi sono dipinti animali e qualche vaso, mentre sul giallo basi di colonne. L'altezza dello zoccolo è di m. 0.55. Il campo basso doveva essere di nero e giallo, mentre quello alto di rosso. Sulla parete ovest, che conserva maggiore quantità di stucco, sul campo alto si nota una prospettiva con due centauri e nel centro un quadro con figure mitologiche. Anche sulla parete sud ed est vi sono avanzi di figure, ma non si distinguono. Presso il pilastro del vano d'ingresso degli ambienti numero 2 e 5 sul pavimento vi è una lastra di marmo bianca larga m. 0.65 , lungo $\mathrm{m}$. 1.10 e profonda $\mathrm{m}$. 0.04 con due buchi al centro che potevano servire come getto d'acqua. La decorazione arrivava fino ai travicelli del compluvio. La muratura è di opera reticolato. Sulla parete ovest vi è un finestrino che serviva per dare la luce al piano superiore esterno e al lato nord una veranda. 
Objets mis au jour dans l'atrium (ambiente 3) :

1040 [ =76317] (08/03/1933): Terracotta. Lucerna monolichne col rostro accorciato. ì lunga m. 0.09 e del diametro di m. 0.05 .

1041 [ =76318] (08/03/1933): Terracotta aretina. Lucerna monolichne del diametro di m. 0.045, lunga m. 0.075..

1042 [ =76319] (08/03/1933) : Terracotta. Lucerna con l'ansa rotta. Misura m. 0.09 di lunghezza e m. 0.05 di diametro.

$1043[=76320](08 / 03 / 1933):$ Terracotta. Bicchiere alto m. 0.058 e del diametro della bocca di m. 0.025 .

1053 [ =76330] (11/03/1933) : Bronzo. Statuetta di Giove nudo. Il braccio destro è teso in avanti. Le dita della stessa mano, due sono metà piegate e le altre aperte. Il braccio sinistro è piegato in alto e la mano è in atto di stringere qualche cosa. (Doveva poggiare su qualche basetta da noi fin oggi non trovata. L'altezza di m. 0.30. È di ottima fattura) [statuette envoyée au MANN le 02/05/1933].

1054 [ =76331] (11/03/1933) : Bronzo. Lucertola lunga m. 0.078.

1055 [ =76332] (11/03/1933) : Bronzo. Lucerna con ansa costolata e rostro accorciato. Il diametro è di m. 0.06 e la lunghezza di m. 0.14.

1059 [ =76336] (19/04/1933) : Bronzo. Cammello che tiene sulla groppa due ceste. All'animale in parola mancano le zampe. La lunghezza è di m. 0.10.

1060 [ =76337] (19/04/1933): Marmo e bronzo. Erma di bronzo sostenuta da base e pilastro di marmo bianco. Il tutto è alto m. 0.29. La testa è di Giove?

$1061[=76338](20 / 04 / 1933):$ Vetro. Unguentario alto m. 0.085 e del diametro di m. 0.015.

$1062[=76339](20 / 04 / 1933):$ Terracotta. Fritillo alto m. 0.87 del diametro della bocca di m. 0.05 .

1063 [ =76340] (20/04/1933) : Bronzo. Pentola rotta ad un lato. È alta m. 0.12 e del diametro di m. 0.12 .

1072 [ =76349] (24/04/1933) : Bronzo. Base di una fruttiera, alta m. 0.20 e della lunghezza di m. 0.22 .

1073 [ =76350] (24/04/1933) : Bronzo. Candelabro mancante della parte superiore. Alto m. 0.185. Inventario n. 1073.

$1081[=76358](09 / 05 / 1933):$ Bronzo. Amorino \{Erote\} alato con basetta. Il tutto è alto m. 0.15. L'Amorino \{Erote\} poggia col piede destro sulla base ed il sinistro lo tiene teso dalla parte posteriore. (Il braccio sinistro è piegato ed il destro è teso in avanti e con la mano stringe un corno. La testa è coperta).

1082 [ =76359] (09/05/1933) : Bronzo. Amorino \{Erote\} alato alto m. 0.138. Poggia col piede sinistro sulla basetta ed il destro è inclinato dalla parte posteriore. (Con la mano destra stringe un corno).

1083 [ =76360] (09/05/1933) : Bronzo. Brucia profumo alto m. 0.08. Il piattello è del diametro di m. 0.075 e profondo m. 0.013 . Il piede è circolare e misura m. 0.05 di diametro. 
1084 [ =76361] (09/05/1933) : Bronzo. Moneta di modulo medio del diametro di m. 0.025. E' dell'epoca di Nerone.

1096 [ =76373] (16/06/1933) : Bronzo. Moneta di modulo medio del diametro di m. 0.025 e dello spessore di m. 0.002 .

1097 [ =76374] (17/06/1933) : Bronzo. Cassarola lunga m. 0.225, ansa compresa e m. 0.115 di diametro.

Sans inventaire (26/04/1933) : Orlo di vaso aretino con le seguenti lettere : ]VII ${ }^{50}$

Sono alte m. 0.01 .

Sans inventaire (02/05/1933) : Parte superiore di un'anfora di terracotta con le seguenti diciture fatte con pittura nera : $\operatorname{Tr}[---] \mid$ Adf $\mid$ Amaxpol $^{51}$

La I ${ }^{a}$ è lunga m. 0.025 ed alta m. 0.015. La II ${ }^{a}$ misura m. 0.04 di lunghezza e m. 0.013 di altezza, e la III ${ }^{\mathrm{a}} \mathrm{m}$. 0.09 di lunghezza e m. 0.015 di altezza.

Sans inventaire (15/05/1933) : Fondo di vaso aretino con il seguente bollo : S(ex) M(urrius) F(estus) [in planta pedis] ${ }^{52}$

E' lungo m. 0.02 ed alto m. 0.004.

Sans inventaire (16/06/1933) : Frammento di tegola di terracotta con il bollo : Hilari $^{53}$

E' spezzata. Le lettere sono alte m. 0.018. La lunghezza attuale è di m. 0.065 .

Pièce 4

1934

9 gennaio. Sul pavimento dell'ambiente n. 10. della casa 7, posta sul IV cardine, lato est, è stato raccolto [Description d'un fragment d'amphore vinaire].

Description générale de la pièce 4 (ambiente 10) : Riceve la luce dal vano che tiene nell'atrio e da una finestra sulla parete ovest [est]. Il vano è largo m. 0.90 ed alto m. 1.90. I pilastri sono di conci di tufo e la soglia di lava vulcanica della lunghezza di m. 0.42. Alle due estremità conserva l'impronta dei cardini che sono a forma quadrata di m. 0.07 per lato. La finestra si sviluppa a m. 1.70 di altezza dal pavimento e misura m. di $0.95 \mathrm{di}$ lunghezza per m. 0.79 di altezza. L'ambiente è largo m. 3.62, lungo m. 2.45 ed alto m. 3.42. La costruzione delle pareti è di opera incerta fatta di frammenti di tufo con qualche rara scoria di lava vulcanica. Il pavimento è di tessere bianche con una fascia di coronamento di tessere nere della larghezza di m. 0.03. Sulla parete est a m. 1.30 di altezza dal suolo vi è dipinta una baccante sdraiata che poggia il gomito sinistro sopra un masso. Dalla cintola in sopra è nuda, mentre tutta la parte bassa è coperta da drappo. Tiene la testa girata verso la sinistra ed il braccio destro disteso. Il tutto è sopra stucco a fondo giallo. Ai lati della baccante, ma più basso, sopra una terrazza chiusa la parte anteriore da una transenna di legno, due donne in piedi completamente vestite che hanno lo sguardo verso terra. Sulle altre pareti accenni di prospettiva su stucco bianco. Lo zoccolo, parte dello stucco è granata parte giallo. Sul campo alto si vedono figure volatili, festoni e prospettive. Termina la parte alta con una cornice di stucco a rilievo con palmette e volute policromate rosso. La cornice è alta $\mathrm{m}$. 0.15 . Il soffitto era fatto a cannucce, ma pochissimo, e tutto marcito, ne fu raccolto.

Objet mis au jour dans la pièce 4 (ambiente 10) : 
Sans inventaire (09/01/1934) : Coccio di terracotta appartenente ad una anfora vinaria con la seguente iscrizione fatta con pittura nera. $\bar{m} a p t^{54}$.

È lunga m. 0.031 ed alta m. 0.025 .

\section{NOTES}

1. Casa di Nettuno ed Anfitrite (insula V), Casa del colonnato tuscanico et Casa del salone nero (insula VI), Casa del tramezzo di legno et Casa dello scheletro (insula III).

2. Dardenay et al. 2015.

3. "La Casa del salone nero à Herculanum», sous la direction d'A. Dardenay, Université de Toulouse II-Jean Jaurès, master 2 soutenu le 10 juin 2015.

4. «La Casa d'Argo dans le contexte de l'insula II d'Herculanum », sous la direction de C. Bonnet et

A. Dardenay, Université de Toulouse II-Jean Jaurès.

5. Ruggiero 1885 , p. 540-561.

6. « Un quadro che esprime Mercurio che tenta di addormentare Argo e rapirgli la bella Io è in un lato di questa stanza ed è circondato da trofei e da decorazioni del più pregevole stile ", selon le rapport de C. Bonucci (Ruggiero 1885, p. 559). Une gravure de ce tableau fut publiée en 1832 dans le volume VIII du Real Museo Borbonico, pl. XXV. Il existe également une mention chez Helbig, n - 136 qui précise que le tableau est détruit.

7. Schefold 1958, p. 353 ; Herbig 1962, p. 13, pl. 23-26.

8. Maiuri 1958, p. 368.

9. Pagano - Prisciandaro 2006, p. 60 avec traduction erronée («ramo intrecciato con tralci e uva »).

10. Allroggen-Bedel 1974, p. 123-124 fig. 6-9.

11. Pagano - Prisciandaro 2006, p. 55.

12. Pagano - Prisciandaro 2006, p. 55 : lunghezza $68,2 \mathrm{~cm}$, altezza $17,6 \mathrm{~cm}$. MANN 9821 : lunghezza $63 \mathrm{~cm}$, altezza $12 \mathrm{~cm}$.

13. Pagano - Prisciandaro 2006, p. 55.

14. Allroggen-Bedel 2009, p. 173-177 fig. 6-9.

15. Ruggiero 1885, p. 560.

16. Ruggiero 1885, p. 561-562.

17. Finati 1839.

18. Voir par exemple Antoni 2009.

19. Le site a également livré des statuettes de terre cuite et d'exceptionnelles sculptures en bois.

20. On pense à l'œuvre éponyme de la Casa dell'erma di bronzo qui rappelle l'hermès pompéien de L. Caecilius Iucundus.

21. Adamo Muscettola 1984 ; Kaufmann-Heinimann 1998, p. 209-226.

22. Voir en particulier l'ensemble de la Casa a Graticcio dont cinq statuettes sont présentées dans Budetta - Pagano 1988 (cat. $n^{\circ} 11-15$, p. 40-49). Sur le contexte de découverte et pour un inventaire complet des dix statuettes découvertes rangées dans un meuble à l'étage, voir Monteix 2010, p. 26-35 et n. 174 p. 30.

23. Cinquantaquattro - Capaldi - Sampaolo 2014, p. 122, cat. $n^{\circ} X I .90$.

24. Sur cette question voir Cadario 2015.

25. Sur ce relief, voir les études successives de Caruso 2011 et Ciotola 2013.

26. Sur ce relief, voir Böhm 2008.

27. Pour l'interprétation, voir Caruso 2011, p. 31-32.

28. Elles sont aujourd'hui conservées au dépôt archéologique d'Herculanum. 
29. CIL IV, 10825, erronément reportée par M. Della Corte $\left(1958\right.$, p. $\left.273, \mathrm{n}^{\circ} 423\right)$ lors de la relecture de ses notes puis par P. Ciprotti dans le volume du Corpus comme provenant de la Casa sannitica $\mathrm{V}, 1-2$.

30. La figure féminine représentée sur le mur oriental est perçue comme une bacchante. Voir infra pour l'interprétation que nous proposons.

31. GSE $1933:$ : 20 aprile. [...]». Nous commentons cette découverte plus bas.

GSE 1934 (texte inséré à la fin du mois de juin) : «[...] Nei pressi dell'ara furono raccolti i due marmi dipinti e la statua di Ercole nudo ». Cette mention d'une statue d'Hercule est fautive, il s'agirait, selon la description en cours de fouille et des inventaires, de Jupiter.

32. Maiuri 1958, p. 394-396.

33. Maiuri 1958, p. 397.

34. Maiuri 1958, fig. 332, p. 396.

35. Niccolini 1896.

36. Vitr., Arch., 7, 10, 1-2 ; Plin. NH 35, 25.

37. Maiuri 1958, p. 397.

38. Nous remercions Domenico Camardo et Mario Notomista pour les informations qu'ils nous ont apportées.

39. Voir la citation en annexe.

40. Bragantini - Sampaolo 2009, p. 119, n $14 a$

41. Maiuri 1958, p.394-396.

42. Pagano 2005, p.73; Antichità d'Ercolano 1757, p. 1, n. 6.

43. De Vos - De Vos 1982, p.293.

44. Guidobaldi 2008 , p. $251, \mathrm{n}^{\circ} 17$.

45. Allroggen-Bedel 1983, p. 154 n. 81.

46. Pagano 2005, p. 73.

47. Archivio di Stato di Napoli, Casa Reale Antica, fascio 1538 Nr. 59 (lettre d'Alcubierre au Marquis Salas). Pagano 2005, p. 73.

48. Allroggen-Bedel 2010, 369.

49. CIL IV 10480. «Casa 6 - Cardo IV - lato E[st] - Amb[iente] 3 - Parete O[vest] Nella cornice d [estra] in alto di un grande quadro leggesi in col[ore] marrone PVR|PVRA [dessin de l'inscription suivant sa forme en équerre] » (Getty Research Institute, H. B. Vander Poel Campanian collection, I.C, box 51, 6, carnet $n^{\circ} 45,20$ mars 1933). Sur cette inscription, voir Dardenay et al. 2015, § 8.

50. CIL IV 10868. « [indication IV, 4 ajoutée au crayon bleu] Casa 6 (Cardo IV - lato E[st]) - Atrio Framm[ento] di tazza aretina, con graffito nella creta fresca (?------)ull » (Getty Research Institute, H. B. Vander Poel Campanian collection, I.C, box 51, 6, carnet $n^{\circ} 45,17$ mai 1933).

51. CIL IV, 10840. « [indication IV, 4 ajoutée au crayon bleu] Casa 6 (IV Card[o] - lato E[st]) - Amb[iente] 3. Due framm[enti] di grande anfora ovoidale a breve collo e brevi anse, con iscr [izione] nera » (Getty Research Institute, H. B. Vander Poel Campanian collection, I.C, box 51, 6, carnet $\mathrm{n}^{\circ} 45,17$ mai 1933).

52. CIL X, 8055, 25 (CVAr II 1212). « Casa 6 (IV Card[o]- lato E[st]) - Amb[iente] 3 nell'atrio fondo di tazza aretina col bollo » (Getty Research Institute, H. B. Vander Poel Campanian collection, I.C, box 51,6 , carnet $\mathrm{n}^{\circ} 45,17$ mai 1933).

53. CIL X, 8074, 6. « [indication IV, 3-4 ajoutée au crayon bleu] IV Card[o]- Lato E[st] - Casa 6 Atrio - Framm[ento] di tegola col bollo a $1[$ ievo] ril[ievo] » (Getty Research Institute, H. B. Vander Poel Campanian collection, I.C, box 51, 6, carnet $\mathrm{n}^{\circ} 46,29$ août 1933).

54. CIL X, 10825. « [indication V, 1 ajoutée au crayon bleu] Card[o] 4 - Casa 7 - Lato E[st] - Framm [ento] d'anfora su cui in col[ore] nero " (Getty Research Institute, H. B. Vander Poel Campanian collection, I.C, box 51, 6, carnet $n^{\circ} 46,10$ janvier 1934). 


\section{INDEX}

institutions Soprintendenza speciale per i beni archeologici di Pompei, Ercolano e Stabia, Ufficio Scavi di Ercolano, Musée archéologique national de Naples, École française de Rome, Centre Jean Bérard, CNRS, ANR, ENS, Université Toulouse-Jean Jaurès

Mots-clés : Herculanum, urbanisme romain, architecture, habitat, décor antique, peinture murale, infographie, 3D

\section{AUTEURS}

\section{ALEXANDRA DARDENAY}

Université Toulouse II Jean Jaurès, UMR 5608 « TRACES » (CNRS / Université de Toulouse II) - adardenay[at]yahoo.fr

\section{AGNES ALLROGGEN-BEDEL}

riess-ab[at]t-online.de

HÉLÈNE ERISTOV

UMR 8546 « AOROC » (CNRS / ENS) - helene.eristov[at]ens.fr

\section{ADELINE GRAND-CLÉMENT}

Université Toulouse Jean Jaurès, PLH-ERASME - adelinegc[at]yahoo.fr

\section{MARIE-LAURE MARAVAL}

Université Toulouse II Le Mirail, DAR (Direction Appui Recherche), UMR 5608 « TRACES »

(CNRS / Université de Toulouse II) - mmaraval[at]univ-tlse2.fr

\section{CARLA MAROTTA}

Université Toulouse Jean Jaurès - carla.marotta90[at]gmail.com

\section{NICOLAS MONTEIX}

Université de Rouen, GRHis (EA 3831) - nicolas.monteix[at]univ-rouen.fr

\section{EMMANUELLE ROSSO}

Université Paris-Sorbonne (Paris IV) - rosso_emma[at]yahoo.fr 